\title{
UNCONDITIONALLY STABLE SCHEMES FOR HIGHER ORDER INPAINTING*
}

\author{
CAROLA-BIBIANE SCHÖNLIEB $^{\dagger}$ AND ANDREA BERTOZZI ${ }^{\ddagger}$
}

\begin{abstract}
Higher order equations, when applied to image inpainting, have certain advantages over second order equations, such as continuation of both edge and intensity information over larger distances. Discretizing a fourth order evolution equation with a brute force method may restrict the time steps to a size up to order $\Delta x^{4}$ where $\Delta x$ denotes the step size of the spatial grid. In this work we present efficient semi-implicit schemes that are guaranteed to be unconditionally stable. We explain the main idea of these schemes and present applications in image processing for inpainting with the Cahn-Hilliard equation, $\mathrm{TV}-\mathrm{H}^{-1}$ inpainting, and inpainting with LCIS (low curvature image simplifiers).
\end{abstract}

Key words. Image inpainting, higher order equations, numerical schemes.

AMS subject classifications. 35G25; 34K28.

\section{Introduction}

An important task in image processing is the process of filling in missing parts of damaged images based on the information gleaned from the surrounding areas. It is essentially a type of interpolation and is called inpainting. Thereby one could restore images with damaged parts due to, for instance, intentional scratching, aging, or weather. Or one can recover objects which are occluded by other objects, where within this context the process is called disocclusion. In fact the applications of image inpainting are countless. From the restoration of ancient frescoes [3], to the medical needs of reducing artifacts in MRI-, CT- or PET imaging reconstructions [47], digital image inpainting is ubiquitous in our modern computerized society. Since the first works on image inpainting by Mumford, Nitzberg and Shiota [57], Masnou and Morel [52], Caselles, Morel, Sbert and Gillette [21], and Bertalmio et al [10], much effort has gone into developing digital algorithms. These methods include the texture synthesis and exemplar-based approach (see, e.g., [20, 29, 32, 72]) and a number of variationaland PDE-based approaches. This paper focuses on the latter.

In mathematical terms, image inpainting can be described in the following way: let $f$ be the given image defined on an image domain $\Omega$. The problem is to reconstruct the original image $u$ in the damaged domain $D \subset \Omega$, called the inpainting domain. More precisely, let $\Omega \subset \mathbb{R}^{2}$ be an open and bounded domain with Lipschitz boundary, $B_{1}, B_{2}$ two Banach spaces and $f \in B_{1}$ be the given image. A general variational approach in inpainting can be written as

$$
\min _{u \in B_{2}}\left\{E(u)=R(u)+\|\lambda(f-u)\|_{B_{1}}^{2}\right\}
$$

where $R: B_{2} \rightarrow \mathbb{R}$ and

$$
\lambda(x)= \begin{cases}\lambda_{0} & \Omega \backslash D \\ 0 & D\end{cases}
$$

${ }^{*}$ Received: September 8, 2009; accepted (in revised version): September 7, 2010. Communicated by Martin Burger.

${ }^{\dagger}$ Institute for Numerical and Applied Mathematics, Georg-August University of Göttingen, Lotzestr. 16-18, D-37083 Göttingen, Germany (c.schoenlieb@math.uni-goettingen.de).

$\ddagger$ Department of Mathematics, UCLA (University of California Los Angeles), 520 Portola Plaza, Los Angeles, CA 90095-1555, USA (bertozzi@math.ucla.edu). 
is the characteristic function of $\Omega \backslash D$ multiplied by a constant $\lambda_{0} \gg 1$. $R(u)$ denotes the regularizing term and $\|\lambda(f-u)\|_{B_{1}}$ the so called fidelity term of the inpainting approach. $B_{2} \subseteq B_{1}$ in general, signifying the smoothing effect of the regularizing term on the minimizer $u \in B_{2}$. Depending on the choice of the regularizing term $R$ and the Banach spaces $B_{1}, B_{2}$, various inpainting approaches have been developed. The most famous model is the total variation (TV) model, where $R(u)=\int_{\Omega}|\nabla u| d x$ denotes the total variation of $u, B_{1}=L^{2}(\Omega)$ and $B_{2}=B V(\Omega)$ the space of functions of bounded variation; $\mathrm{cf}$. $[23,25,61,60]$. A variational model with a regularizing term containing higher order derivatives is the Eulers elastica model $[26,27,52]$ where $R(u)=\int_{\Omega}(a+$ $\left.b \kappa^{2}\right)|\nabla u| d x$ with positive weights $a$ and $b$, and curvature $\kappa=\nabla \cdot(\nabla u /|\nabla u|)$. Other examples to be mentioned for (1.1) are the active contour model based on Mumford and Shahs segmentation [68], the inpainting scheme based on the Mumford-Shah-Euler image model [35], inpainting with the Navier-Stokes equation [11], and wavelet-based inpainting $[28,30]$, only to give a rough overview. For a more complete introduction to image inpainting using PDEs we refer to $[26,18,63]$.

1.1. Second- versus higher-order inpainting approaches. Second order variational inpainting methods (where the order of the method is determined by the derivatives of highest order in the corresponding Euler-Lagrange equation), like TV inpainting, have drawbacks as in the connection of edges over large distances (Connectivity Principle, cf. Figure 1.1) and the smooth propagation of level lines (sets of image points with constant grayvalue) into the damaged domain (Curvature Preservation, cf. Figure 1.2). This is due to the penalization of the length of the level lines within the minimizing process with a second order regularizer, connecting level lines from the boundary of the inpainting domain via the shortest distance (linear interpolation). The regularizing term $R(u)=\int_{\Omega}|\nabla u| d x$ in the TV inpainting approach, for example, can be interpreted via the coarea formula, which gives

$$
\min _{u} \int_{\Omega}|\nabla u| d x \Longleftrightarrow \min _{\Gamma_{\lambda}} \int_{-\infty}^{\infty} \operatorname{length}\left(\Gamma_{\lambda}\right) d \lambda
$$

where $\Gamma_{\lambda}=\{x \in \Omega: u(x)=\lambda\}$ is the level line for the grayvalue $\lambda$. If we consider on the other hand the regularizing term in the Eulers elastica inpainting approach the coarea formula reads

$$
\min _{u} \int_{\Omega}\left(a+b \kappa^{2}\right)|\nabla u| d x \Longleftrightarrow \min _{\Gamma_{\lambda}} \int_{-\infty}^{\infty} a \operatorname{length}\left(\Gamma_{\lambda}\right)+b \operatorname{curvature}^{2}\left(\Gamma_{\lambda}\right) d \lambda .
$$

Thus not only the length of the level lines but also their curvature is penalized (where the penalization of each depends on the ratio $b / a$ ). This results in a smooth continuation of level lines over the inpainting domain also over large distances; compare Figures 1.1 and 1.2. The performance of higher order inpainting methods can also be interpreted via the second boundary condition, which is necessary for the wellposedness of the corresponding Euler-Lagrange equation of fourth order. Not only are the grayvalues of the image specified on the boundary of the inpainting domain, but also the gradient of the image function, namely the direction of the level lines, is given.

In an attempt to solve both the connectivity principle and the staircasing effect resulting from second order image diffusions, a number of third and fourth order diffusions have been suggested for image inpainting. The first work connecting image inpainting to a third order PDE (partial differential equation) is the transport process 

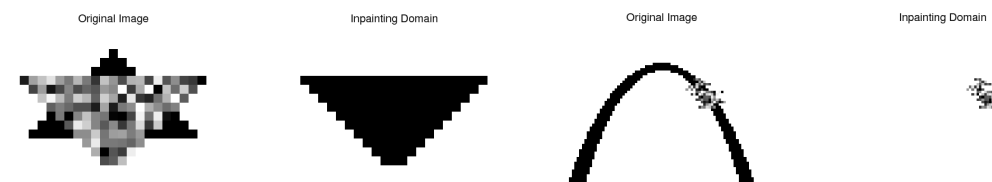

T:
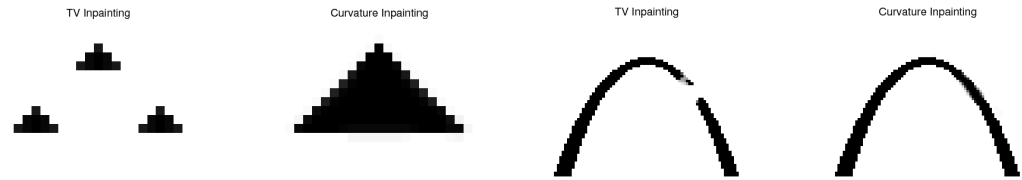

FIG. 1.1. Two examples of curvature based inpainting compared with TV inpainting from [26]. In the case of large aspect ratios the TV inpainting fails to comply to the Connectivity Principle.
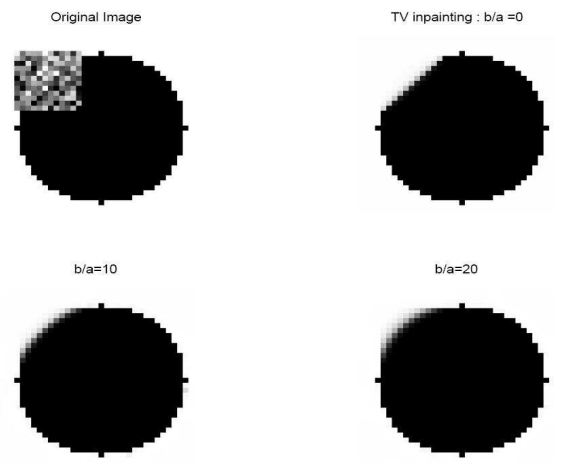

FIG. 1.2. An example of elastica inpainting compared with TV inpainting from [27]. Despite the presence of high curvature, TV inpainting truncates the circle inside the inpainting domain (linear interpolation of level lines, i.e., Curvature Preservation). Depending on the weights a and $b$ Eulers elastica inpainting returns a smoothly restored object, taking the curvature of the circle into account.

of Bertalmio et al [10]. The image information, modeled by $\Delta u$, is transported into the inpainting domain along the level lines of the image. The resulting scheme is a discrete model based on the nonlinear PDE

$$
u_{t}=\nabla^{\perp} u \cdot \nabla \Delta u,
$$

and is solved inside the inpainting domain $D$ using the image information from a small stripe around the boundary of $D$. The operator $\nabla^{\perp}$ denotes the perpendicular gradient $\left(-\partial_{y}, \partial_{x}\right)$. Due to the lack of communication among the level lines, the transportation may result in kinks or contradictions inside the inpainting domain. Thus in [10] the equation above is implemented with intermediate steps of anisotropic diffusion. In [11] the authors develop a theory for the proper boundary conditions in [10] by making a connection to the Navier-Stokes equations. The two conditions on the "boundary" of the inpainting domain correspond to the no slip condition for NavierStokes. A variational third order approach to image inpainting is CDD (Curvature Driven Diffusion) [24]. To solve the problem of connecting level lines also over large 
distances (connectivity principle), the level lines are still interpolated linearly. The drawbacks of the third-order inpainting models [10] and [24] have driven Chan, Kang and Shen [27] to a reinvestigation of the earlier proposal of Masnou and Morel [52] on image interpolation based on Eulers elastica energy (1.3). The fourth order elastica inpainting PDE combines CDD [24] and the transport process of Bertalmio et al [10], and is able to solve both the connectivity principle and the staircasing effect. Other recently proposed higher order inpainting algorithms are inpainting with the CahnHilliard equation [13, 14], TV-H ${ }^{-1}$ inpainting $[19,64]$ and combinations of second and higher order methods, e.g. [51].

In this paper we are especially interested in three, rather new, fourth-order inpainting schemes. Namely, we shall discuss Cahn-Hilliard inpainting, $\mathrm{TV}^{-} \mathrm{H}^{-1}$ inpainting, and inpainting with LCIS (low curvature image simplifiers). We start the discussion with the inpainting of binary images using the Cahn-Hilliard equation [13, 14]. The inpainted version $u$ of $f \in L^{2}(\Omega)$ is constructed by following the evolution of

$$
u_{t}=\Delta\left(-\epsilon \Delta u+\frac{1}{\epsilon} F^{\prime}(u)\right)+\lambda(f-u)
$$

where $F(u)$ is a so called double-well potential, e.g., $F(u)=u^{2}(u-1)^{2}$. The applicability of the Cahn-Hilliard equation for the inpainting of binary images is due to the double well potential $F(u)$ in the equation. The two wells correspond to values of $u$ that are taken by most of the grayscale values. Choosing a potential with wells at the values 0 (black) and 1 (white), Equation (1.4) therefore provides a simple model for the inpainting of binary images. The parameter $\epsilon$ determines the steepness of the transition between 0 and 1 . Further, the fourth order regularizing term in the equation provides the advantages of higher order inpainting approaches which have been discussed before, such as the ability to connect level lines also over large distances (cf. $(1.3))$.

The second method of interest in this paper is a generalization of the CahnHilliard inpainting approach to grayvalue images which has been recently proposed in $[19,64]$ and is called $T V-H^{-1}$ inpainting. Therein the inpainted image $u$ of $f \in L^{2}(\Omega)$ shall evolve via

$$
u_{t}=\Delta p+\lambda(f-u), \quad p \in \partial T V(u)
$$

with

$$
T V(u)= \begin{cases}\int_{\Omega}|\nabla u| d x, & \text { if }|u(x)| \leq 1 \text { a.e. in } \Omega, \\ +\infty, & \text { otherwise, }\end{cases}
$$

where $\partial T V(u)$ denotes the subdifferential of the functional $T V(u)$. To build the connection to Cahn-Hilliard inpainting the authors in [19] show that solutions of an appropriate time-discrete Cahn-Hilliard inpainting approach $\Gamma$-converge, as $\epsilon \rightarrow 0$, to solutions of an optimization problem regularized with the TV norm. A similar form of this approach appears in the context of decomposition and restoration of grayvalue images; see for example [49, 58, 70]. Further, in Bertalmio et al [12], an application of the model from [70] to image inpainting is proposed. In contrast to the inpainting approach (1.5) the authors in [12] use a more general form of the TV- $\mathrm{H}^{-1}$ approach for a decomposition of the image into cartoon and texture prior to the inpainting process. The latter is accomplished with the method presented in [10]. Moreover, we 
would like to mention that in [45] the authors consider a complex Ginzburg-Landau energy for inpainting of grayscale- and color images.

The third inpainting model we are going to discuss is inpainting with LCIS (Low Curvature Image Simplifier). This higher order inpainting model is motivated by two famous second order nonlinear PDEs in image processing - the works of Rudin, Osher and Fatemi [60] and Perona Malik [59]. These methods are based on a nonlinear version of the heat equation

$$
u_{t}=\nabla \cdot(g(|\nabla u|) \nabla u),
$$

in which $g$ is small in regions of sharp gradients. LCIS represent a fourth order relative of these nonlinear second order approaches. They are proposed in [69] and later used by Bertozzi and Greer in [15] for the denoising of piecewise linear signals. In this paper we consider LCIS for image inpainting. With $f \in L^{2}(\Omega)$ our inpainted image $u$ evolves in time as

$$
u_{t}=-\nabla \cdot(g(\Delta u) \nabla \Delta u)+\lambda(f-u),
$$

with thresholding function $g(s)=\frac{1}{1+s^{2}}$. Note that with $g(\Delta u) \nabla \Delta u=\nabla(\arctan (\Delta u))$ the above equation can be rewritten as

$$
u_{t}=-\Delta(\arctan (\Delta u))+\lambda(f-u) .
$$

\subsection{Numerical solution of higher-order inpainting equations.}

One main challenge in inpainting with higher order flows is their effective numerical implementation. Discretizing a fourth order evolution equation with a brute-force method may restrict the time steps to a size up to order $\Delta x^{4}$ where $\Delta x$ denotes the step size of the spatial grid. Such a brute-force method is computationally prohibitive and hence it is essentially never done; see, e.g., [65].

The numerical solution of higher-order equations, like thin films, phase field models, surface diffusion equations, and many more, occupied a big part of research in numerical analysis in the last decades. In [31] the authors propose a semi implicit finite difference scheme for the solution of second order parabolic equations. A diffusion term is added implicitly and subtracted explicitly in time to the numerical scheme in order to suppress unstable modes. Smereka uses this idea to solve the fourth-order surface diffusion equation; cf. [65]. The same idea is applied by Glasner to a phase field approach for the Hele-Shaw interface model; cf. [40]. Besides the finite difference approximations, there also exist many finite element algorithms for fourth-order equations. Barrett, Blowey, and Garcke published a series of papers on the solution of various Cahn-Hilliard equations; cf. [5, 6, 7]. For the sharp interface limit of CahnHilliard, i.e., the Hele-Shaw model, Feng and Prohl analyze finite element methods in $[37,38]$. Finite element methods for thin film equations are studied, for instance, in $[8,46]$.

For image inpainting, efficient numerical schemes for higher-order methods is an active area of research. As discussed in [26] one of the most interesting open problems in digital inpainting is, in fact, the fast and exact digital realization. In the case of Cahn-Hilliard inpainting, in [13] the authors propose a semi-implicit scheme which constitutes the common numerical method discussed in this paper. They verify its computational superiority compared with currently used numerical methods for three curvature driven approaches. It turns out that Cahn-Hilliard inpainting performs at least one order of magnitude faster than the curvature methods. In [33, 34] Elliott 
and Smitheman propose a finite element method for $\mathrm{TV}-\mathrm{H}^{-1}$ minimization in the context of image denoising and cartoon/texture decomposition. They also prove rigorous results about the approximation and convergence properties of their scheme. An extension of their approach to $\mathrm{TV}-\mathrm{H}^{-1}$ inpainting would be interesting. Note that, however, the difference of the inpainting approach from denoising and decomposition is that the former does not follow a variational principle and the fidelity term is locally dependent on the spatial position. Another algorithm for TV-H ${ }^{-1}$ inpainting is proposed by one of the authors in [62]. This work generalizes the dual approach of Chambolle [22] and Bect et al. [9] from an $L^{2}$ fidelity term to an $\mathrm{H}^{-1}$ fidelity and extends its application from $\mathrm{TV}-\mathrm{H}^{-1}$ denoising $[1,2]$ to image inpainting. The main motivation for the work in [62] is that with the proposed algorithm the domain decomposition approach developed in [39] can be applied to the higher-order total variation case. Being able to apply domain decomposition methods to $\mathrm{TV}-\mathrm{H}^{-1}$ inpainting can result in a tremendous acceleration of computational speed due to the ability to parallelize the computation. Another very recent approach in this direction is [18], where the authors propose a multigrid approach for inpainting with CDD.

In this paper we discuss an efficient semi implicit approach based on a numerical method presented in Eyre [36] (also cf. [71]) called convexity splitting. Convexity splitting was originally proposed to solve energy minimizing equations. We consider the following problem: Let $E \in C^{2}\left(\mathbb{R}^{N}, \mathbb{R}\right)$ be a smooth functional from $\mathbb{R}^{N}$ into $\mathbb{R}$, where $N$ is the dimension of the data space. Let $\Omega$ be the spatial domain of the data space. Find $u \in \mathbb{R}^{N}$ such that

$$
\begin{cases}u_{t}=-\nabla E(u), & \text { in } \Omega, \\ u(., t=0)=u_{0}, & \text { in } \Omega,\end{cases}
$$

with initial condition $u_{0} \in \mathbb{R}^{N}$. The basic idea of convexity splitting is to split the functional $E$ into a convex and a concave part. In the semi implicit scheme, the convex part is treated implicitly and the concave one explicitly in time. Under additional assumptions on (1.7), this discretization approach is unconditionally stable, consistent, and relatively easy to apply to a large range of variational problems. Moreover we shall see that the idea of convexity splitting can be applied to more general evolution equations, and in particular to those that do not follow a variational principle, especially to the inpainting Equations (1.4) and (1.5).

Convexity splitting methods, although possibly not under the same name, already have a long tradition in several parts of numerical analysis. In finite element approximations for PDEs, examples for such numerical schemes can be found in the works of Barrett, Blowley, and Garcke; cf. [4] Equation (3.42) for an application to a model for phase separation. In [35] a finite difference scheme for second-order parabolic equations is presented which also uses the convexity splitting idea; cf. Equation (5.4) in [35]. Further convexity splitting is also discussed in a more general optimization context; cf. [73] Chapter two for an overview on this topic.

The main part of the paper illustrates the application of the convexity splitting idea to the three fourth-order inpainting approaches (1.4), (1.5), and (1.6). Motivated by the analysis in [17], we show that with this numerical approach we are able to (approximately) compute strong solutions of the continuous problem with an unconditionally stable finite difference scheme. The numerical scheme is said to be unconditionally stable if all solutions of the difference equation are bounded independently from the time step size; cf. Definition 2.2. Moreover, we prove consistency of these schemes and convergence to the exact solution. Further, we present nu- 
merical results demonstrating the effect of the higher order regularizing term in the approaches. In the case of $\mathrm{TV}-\mathrm{H}^{-1}$ inpainting and inpainting with LCIS we directly compare the visual results with the second order TV inpainting method.

Organization of the paper. In Section 2 the idea of convexity splitting is presented. After an introduction to gradient systems we state and prove Eyre's theorem about the unconditional stability of the convexity splitting scheme. Sections 3-5 are dedicated to the application of convexity splitting to Cahn-Hilliard inpainting (1.4), TV-H ${ }^{-1}$ inpainting (1.5), and inpainting with LCIS (1.6). In the case of Cahn-Hilliard and $\mathrm{TV}-\mathrm{H}^{-1}$ inpainting the corresponding Equations (1.4) and (1.5) are not strictly gradient flows, but their evolution is the sum of the gradients of two different energies. Here, convexity splitting is applied to each of these energies and results in a semi-implicit scheme for the whole evolution. Rigorous proofs for the consistency of the numerical scheme, the boundedness of numerical solutions and their convergence to the exact solution are given. For each of these inpainting algorithms numerical results are presented. In the conclusion of the paper open problems are discussed.

Notation. In this paper we discuss the numerical solution of evolutionary differential equations. Therefore we have to distinguish between the exact solution $u$ of the continuous equation and the approximative solution $U$ of the corresponding time discrete numerical scheme. We write capital $U_{k}$ for the $k_{t h}$ solution of the discrete equation and small $u_{k}=u(k \Delta t)$ for a solution of the continuous inpainting equation at time $k \Delta t$ with time step size $\Delta t$. Let $e_{k}$ denote the temporal discretization error given by $e_{k}=u_{k}-U_{k}$. In subsection two, $u$ and $U$ are vectors in $\mathbb{R}^{N}$, where $N$ denotes the dimension of the data. In all other parts of this paper $u$ and $U$ are assumed to be elements in $L^{2}(\Omega)$. Let $E \in C^{2}(\mathcal{H}, \mathbb{R})$ denote a functional from a suitable Hilbert space $\mathcal{H}$ to $\mathbb{R}$, and $\nabla E(u)$ its first variation with respect to $u$. In the discrete setting $\mathcal{H}=\mathbb{R}^{N}$. Throughout this paper $\|\cdot\|$ denotes the norm in $L^{2}(\Omega)$ (or the Euclidean norm in the discrete setting), and $\langle\cdot, \cdot\rangle$ the inner product in $L^{2}(\Omega)$ (or in $\mathbb{R}^{N}$ in the discrete setting). Finally, since we pose all three inpainting approaches (1.4)-(1.6) with Neumann boundary conditions, we have to define the non-standard space $H^{-1}(\Omega)$ as

$$
H^{-1}(\Omega)=\left\{F \in H^{1}(\Omega)^{*} \mid\langle F, 1\rangle_{\left(H^{1}\right)^{*}, H^{1}}=0\right\},
$$

with norm $\|\cdot\|_{-1}:=\left\|\nabla \Delta^{-1} \cdot\right\|_{L^{2}(\Omega)}$. Thereby the operator $\Delta^{-1}$ denotes the inverse of $\Delta$ with Neumann boundary conditions. In more detail, let $\stackrel{\circ}{H^{1}}(\Omega):=$ $\left\{\psi \in H^{1}(\Omega): \int_{\Omega} \psi d x=0\right\}$. Then $u=\Delta^{-1} F \in \stackrel{\circ}{H^{1}}(\Omega)$ is the unique weak solution of the following problem:

$$
\left\{\begin{array}{l}
\Delta u-F=0, \text { in } \Omega \\
\nabla u \cdot \nu=0, \quad \text { on } \partial \Omega
\end{array}\right.
$$

For a more elaborate derivation of the above space we refer to [19], Appendix A.

\section{The convexity splitting idea}

As already discussed in the Introduction, convexity splitting methods are used in a wide range of optimization problems; cf. Section 1.2 for relevant references. Originally designed to solve gradient systems, we shall see in this paper that convexity splitting schemes are relevant for more general problems, i.e., for evolution equations which do not follow a variational principle. See Sections 3-5 for our three inpainting 
approaches (1.4)-(1.6).

First we introduce the notion of gradient flows and the application of convexity splitting methods in this context. To do so we follow the explanations and notations in Eyre's work [36].

We consider Equation (1.7). If $E$ fulfills the following conditions;

$$
\begin{aligned}
& \text { (i) } E(u) \geq 0, \forall u \in \mathbb{R}^{N}, \\
& \text { (ii) } E(u) \rightarrow \infty \text { as }\|u\| \rightarrow \infty, \\
& \text { (iii) }\langle J(\nabla E)(u) u, u\rangle \geq \lambda \forall u \in \mathbb{R}^{N},
\end{aligned}
$$

then Equation (1.7) is called a gradient system and its solutions are called gradient flows. Thereby $J(\nabla E)(u)$ is the Jacobian of $\nabla E$ in $u, \lambda \in \mathbb{R}$ and $\langle.,$.$\rangle denotes the inner$ product on $\mathbb{R}^{N}$ with corresponding norm $\|u\|^{2}=\langle u, u\rangle$. All gradient systems satisfy the dissipation property, i.e.,

$$
\frac{d E(u)}{d t}=-\|\nabla E(u)\|^{2},
$$

and therefore $E(u(t)) \leq E\left(u_{0}\right)$ for all $t \geq 0$.

If $E(u)$ is strictly convex, i.e., $\lambda$ in condition (2.1)(iii) is positive, then only a single equilibrium for the gradient system exists. Unconditionally stable and uniquely solvable numerical schemes exist for these equations (cf. [66]). If $E(u)$ is not strictly convex, i.e., $\lambda<0$, multiple minimizers may exist and the gradient flow can possibly expand in $u(t)$. The stability of an explicit gradient descent algorithm, i.e., $U_{k+1}=$ $U_{k}-\Delta t \nabla E\left(U_{k}\right)$, in this case may require extremely small time steps, depending of course on the functional $E$. For fourth order inpainting approaches, for instance, $E\left(U_{k}\right)$ contains second order derivatives resulting in a restriction of $\Delta t$ up to order $(\Delta x)^{4}$ (where $\Delta x$ denotes the step size of the spatial discretization). Therefore the development of stable and efficient discretizations for non-convex functionals $E$ is highly desirable.

The basic idea of convexity splitting is to write the functional $E$ as

$$
E(u)=E_{c}(u)-E_{e}(u),
$$

where

$$
E_{o} \in C^{2}\left(\mathbb{R}^{N}, \mathbb{R}\right) \text { and } E_{o}(u) \text { is strictly convex for all } u \in \mathbb{R}^{N}, o \in\{c, e\} .
$$

The semi-implicit discretization of (1.7) is then given by

$$
U_{k+1}-U_{k}=-\Delta t\left(\nabla E_{c}\left(U_{k+1}\right)-\nabla E_{e}\left(U_{k}\right)\right),
$$

where $U_{0}=u_{0}$.

REMARK 2.1. We want to anticipate that the setting of Eyre, and hence the subsequent presentation of convexity splitting, is a purely discrete one. Nevertheless it actually holds in a more general framework, i.e., for more general gradient flows. In the case of an $L^{2}$ gradient flow for example, the Jacobian $J$ of the discrete functional $E$ just has to be replaced by the second variation of the continuous functional $E$ in $L^{2}(\Omega)$.

In the following we show that convexity splitting can be applied to the inpainting approaches (1.4), (1.5), and (1.6), and produces unconditionally gradient stable or unconditionally stable numerical schemes. 
Definition 2.1. [36] A one-step numerical integration scheme is unconditionally gradient stable if there exists a function $E():. \mathbb{R}^{N} \rightarrow \mathbb{R}$ such that, for all $\Delta t>0$ and for all initial data:

(i) $E(U) \geq 0$ for all $U \in \mathbb{R}^{N}$,

(ii) $E(U) \rightarrow \infty$ as $\|U\| \rightarrow \infty$,

(iii) $E\left(U_{k+1}\right) \leq E\left(U_{k}\right)$ for all $U_{k} \in \mathbb{R}^{N}$,

(iv) If $E\left(U_{k}\right)=E\left(U_{0}\right)$ for all $k \geq 0$ then $U_{0}$ is a zero of $\nabla E$ for (1.7) and (2.1).

Note that Cahn-Hilliard inpainting (1.4) and $\mathrm{TV}-\mathrm{H}^{-1}$ inpainting (1.5) are not given by gradient flows. Hence, in the context of these inpainting models the meaning of unconditional stability has to be redefined. Namely, in the case of an evolution equation which does not follow a gradient flow, a corresponding discrete time stepping scheme is said to be unconditionally stable if solutions of the difference equation are bounded within a finite time interval, independently of the step size $\Delta t$.

Definition 2.2. Let $u$ be an element of a suitable function space $\mathcal{H}$ defined on $\Omega \times[0, T]$, with $\Omega \subset \mathbb{R}^{2}$ open and bounded, and $T>0$. Let further $G$ be a real valued function and $u_{t}=G\left(u, D^{\alpha} u\right)$ be a partial differential equation with space derivatives $D^{\alpha} u, \alpha=1, \ldots, 4$. A corresponding discrete time stepping method

$$
U_{k+1}=U_{k}+\Delta t G_{k}\left(U_{k}, U_{k+1}, D^{\alpha} U_{k}, D^{\alpha} U_{k+1}\right),
$$

where $G_{k}$ is a suitable approximation of $G$ in $U_{k}$ and $U_{k+1}$ is

- unconditionally stable, if all solutions of (2.5) are bounded for all $\Delta t>0$ and all $k$ such that $k \Delta t \leq T$.

- consistent if

$$
\lim _{\Delta t \rightarrow 0} \tau_{k}(\Delta t)=0
$$

where $\tau_{k}(\Delta t)$ is the local truncation error of the scheme and defined as

$$
\tau_{k}(\Delta t)=\frac{u_{k+1}-u_{k}}{\Delta t}-G_{k}\left(u_{k}, u_{k+1}, D^{\alpha} u_{k}, D^{\alpha} u_{k+1}\right),
$$

and $u_{k}=u(k \Delta t)$ is the exact solution at time $t=k \Delta t$. In what follows we abbreviate $\tau_{k}$ for $\tau_{k}(\Delta t)$. Moreover, we define the global truncation error to be

$$
\tau(\Delta t)=\max _{k}\left\|\tau_{k}(\Delta t)\right\|_{\mathcal{H}}
$$

A numerical scheme is said to be of order $\mathbf{p}$ in time if

$$
\tau(\Delta t)=O\left(\Delta t^{p}\right) \text { for } \Delta t \rightarrow 0 .
$$

We start with a theorem of Eyre [36]. The proof presented below follows the same arguments as in [36] with additional details.

Theorem 2.3 ([36] Theorem 1). Let E satisfy (2.1), and $E_{c}$ and $E_{e}$ satisfy (2.2)-(2.3). If $E_{e}(u)$ additionally satisfies

$$
\left\langle J\left(\nabla E_{e}\right)(u) u, u\right\rangle \geq-\lambda
$$


when $\lambda<0$ in (2.1)(iii), then for any initial condition the numerical scheme (2.4) is consistent with (1.7), gradient stable for all $\Delta t>0$, and possesses a unique solution for each time step. The local truncation error for each step is

$$
\tau_{k}=\frac{\Delta t}{2}\left(J\left(\nabla E_{c}(\hat{u})\right)+J\left(\nabla E_{e}(\hat{u})\right)\right) \nabla E(u(\xi)),
$$

for some $\xi \in(k \Delta t,(k+1) \Delta t)$ and for some $\hat{u}$ in the parallelopiped with opposite vertices at $U_{k}$ and $U_{k+1}$.

Remark 2.2. Condition (2.7) in Theorem 2.3 is equivalent to the requirement that all the eigenvalues of $J\left(\nabla E_{e}\right)$ dominate the largest eigenvalue $-\lambda$ of $-J(\nabla E)$, i.e.,

$$
\left\langle J\left(\nabla E_{e}\right)(u) u, u\right\rangle \stackrel{(2.7)}{\geq}-\lambda \stackrel{(2.1)}{\geq}\langle-J(\nabla E)(u) u, u\rangle
$$

for all $u \in \mathbb{R}^{N}$, or

$$
\hat{\lambda} \geq|\lambda|, \quad \text { for all eigenvalues } \hat{\lambda}>0 \text { of } E_{e} \text {. }
$$

Proof. (Eyre [36]). The unconditional gradient stability of (2.4) in the sense of Definition 2.1 is established first. By our assumptions in (2.1) properties (i) and (ii) in Definition 2.1 immediately follow. Property (iv) follows from the general behavior of gradient systems, i.e., if $E\left(U_{k}\right)=E\left(U_{0}\right)$ for all $k \geq 0$ then $U_{0}$ is an $\omega$ - limit point of (1.7) and (2.1) and hence $U_{0}$ is a zero of $\nabla E$ (cf. [48]). The main part of the proof consists of the verification of property (iii). Namely we have to show that

$$
E\left(U_{k+1}\right) \leq E\left(U_{k}\right), \quad \forall U_{k} \in \mathbb{R}^{N} .
$$

To do so we consider the difference $E\left(U_{k+1}\right)-E\left(U_{k}\right)$. The proof is by repeated application of Taylor's theorem. We start with an exact expansion of $E$ about $U_{k+1}$ up to second order and obtain

$$
\begin{aligned}
E\left(U_{k}\right)=E\left(U_{k+1}\right)-\langle\nabla & \left.E\left(U_{k+1}\right), U_{k+1}-U_{k}\right\rangle \\
& +\frac{1}{2}\left\langle J\left(\nabla E\left(U_{k+1}-\alpha\left(U_{k+1}-U_{k}\right)\right)\right) U_{k+1}-U_{k}, U_{k+1}-U_{k}\right\rangle
\end{aligned}
$$

for some $\alpha \in(0,1)$. Then by assumption (iii) in (2.1) we get

$$
E\left(U_{k+1}\right)-E\left(U_{k}\right) \leq\left\langle\nabla E\left(U_{k+1}\right), U_{k+1}-U_{k}\right\rangle+|\lambda|\left\|U_{k+1}-U_{k}\right\|^{2} .
$$

By (2.2) and (2.4) this is the same as

$$
\begin{aligned}
E\left(U_{k+1}\right)-E\left(U_{k}\right) \leq & \left\langle\nabla E_{c}\left(U_{k+1}\right)-\nabla E_{e}\left(U_{k+1}\right), U_{k+1}-U_{k}\right\rangle+|\lambda|\left\|U_{k+1}-U_{k}\right\|^{2} \\
& -\left\langle\frac{1}{\Delta t}\left(U_{k+1}-U_{k}\right)+\nabla E_{c}\left(U_{k+1}\right)-\nabla E_{e}\left(U_{k}\right), U_{k+1}-U_{k}\right\rangle \\
= & -\left\langle\nabla E_{e}\left(U_{k+1}\right)-\nabla E_{e}\left(U_{k}\right), U_{k+1}-U_{k}\right\rangle+\left(|\lambda|-\frac{1}{\Delta t}\right)\left\|U_{k+1}-U_{k}\right\|^{2} .
\end{aligned}
$$

Similarly, we Taylor expand $E_{e}$ about $U_{k+1}$ and $U_{k}$, respectively, as

$$
\begin{aligned}
E_{e}\left(U_{k}\right)= & E_{e}\left(U_{k+1}\right)-\left\langle\nabla E_{e}\left(U_{k+1}\right), U_{k+1}-U_{k}\right\rangle \\
& +\frac{1}{2}\left\langle J\left(\nabla E_{e}\left(U_{k+1}-\alpha_{1}\left(U_{k+1}-U_{k}\right)\right)\right) U_{k+1}-U_{k}, U_{k+1}-U_{k}\right\rangle,
\end{aligned}
$$


and

$$
\begin{aligned}
E_{e}\left(U_{k+1}\right)=E_{e}\left(U_{k}\right)+\langle & \left.\nabla E_{e}\left(U_{k}\right), U_{k+1}-U_{k}\right\rangle \\
& +\frac{1}{2}\left\langle J\left(\nabla E_{e}\left(U_{k}-\alpha_{2}\left(U_{k+1}-U_{k}\right)\right)\right) U_{k+1}-U_{k}, U_{k+1}-U_{k}\right\rangle,
\end{aligned}
$$

for some $\alpha_{1}$ and $\alpha_{2}$ in $(0,1)$. Since $E_{e}$ is convex, then $J\left(\nabla E_{e}\right)$ is positive definite and its eigenvalues are positive. By bounding the eigenvalues of $J\left(\nabla E_{e}\right)$ by $\hat{\lambda}>0$ and adding the above expressions we get

$$
\left\langle\nabla E_{e}\left(U_{k+1}\right)-\nabla E_{e}\left(U_{k}\right), U_{k+1}-U_{k}\right\rangle \geq \hat{\lambda}\left\|U_{k+1}-U_{k}\right\|^{2} .
$$

Substituting this in (2.9), we obtain

$$
E\left(U_{k+1}\right)-E\left(U_{k}\right) \leq-\left(\hat{\lambda}-|\lambda|+\frac{1}{\Delta t}\right)\left\|U_{k+1}-U_{k}\right\|^{2} .
$$

By applying condition (2.7) (i.e., (2.8)) the result follows for all $\Delta t \geq 0$. Hence the method is unconditionally gradient stable.

To prove the unique solvability of (2.4) we consider the nonlinear equations

$$
U_{k+1}+\Delta t \nabla E_{c}\left(U_{k+1}\right)=R_{k},
$$

which must be solved at each step for a given $R_{k}$. Since $E_{c}$ is strictly convex,

$$
\frac{1}{2}\left\|U_{k+1}\right\|^{2}+\Delta t E_{c}\left(U_{k+1}\right)-\left\langle U_{k+1}, R_{k}\right\rangle
$$

has a unique minimum in $U_{k+1}$ for all $\Delta t$, and (2.4) has a unique solution for all $\Delta t \geq 0$. The consistency and the local truncation error of (2.4) can be established by similar Taylor expansions as the ones we did above to prove the unconditional stability of the scheme. More precisely it consists of expanding $U_{k+1}$ and $U_{k}$ around $(k+1 / 2) \Delta t$, and $\nabla E_{c}\left(U_{k+1}\right)$ and $\nabla E_{e}\left(U_{k}\right)$ around $U_{k+1 / 2}$. This finishes the proof of Theorem 2.3.

In the following we apply the idea of convexity splitting to our three inpainting models (1.4), (1.5), and (1.6). For this we change from the discrete setting to the continuous setting, i.e., considering functions $u$ in a suitable Hilbert space instead of vectors $u$ in $\mathbb{R}^{N}$. Although the first two of these inpainting approaches, i.e., CahnHilliard inpainting and $\mathrm{TV}-\mathrm{H}^{-1}$ inpainting, are not given by gradient flows, we show that the resulting numerical schemes are still unconditionally stable (in the sense of Definition 2.2) and therefore suitable to solve them accurately and reasonably fast. For inpainting with LCIS (1.6) the results of Eyre can be directly applied, even in the continuous setting; cf. Remark 2.1. Nevertheless, also for this case, we additionally present a rigorous analysis, similar to the one done for Cahn-Hilliard and $\mathrm{TV}-\mathrm{H}^{-1}$ inpainting.

\section{Cahn-Hilliard inpainting}

In this section we show the application of convexity splitting to Cahn-Hilliard inpainting (1.4). Recall that the inpainted version $u(x)$ of $f(x)$ is constructed by following the evolution equation

$$
u_{t}=\Delta\left(-\epsilon \Delta u+\frac{1}{\epsilon} F^{\prime}(u)\right)+\lambda(f-u)
$$


to steady state. This modified Cahn-Hilliard equation is introduced in [13] for the inpainting of binary images. The latter, mainly numerical paper, was followed by a very careful analysis of (1.4) in [14]. To start with, the authors prove global existence of a unique weak solution of the evolution Equation (1.4). More precisely the solution $u$ is proven to be an element in $C\left([0, T] ; L^{2}(\Omega)\right) \cap L^{2}(0, T ; V)$, where $V=\left\{\phi \in H^{2}(\Omega) \mid \partial \phi / \partial \nu=0\right.$ on $\left.\partial \Omega\right\}$, and $\nu$ is the outward pointing normal on $\partial \Omega$. Under additional conditions on the given image $f$, they also derive some very interesting results concerning the continuation of the gradient of the image into the inpainting domain. In fact, in [14] the authors prove that in the limit $\lambda_{0} \rightarrow \infty$ a stationary solution of (1.4) solves

$$
\begin{aligned}
\Delta\left(\epsilon \Delta u-\frac{1}{\epsilon} F^{\prime}(u)\right) & =0, & & \text { in } D, \\
u & =f, & & \text { on } \partial D, \\
\nabla u & =\nabla f, & & \text { on } \partial D,
\end{aligned}
$$

for $f$ regular enough $\left(f \in C^{2}\right)$. The existence of a stationary solution of (1.4) is assured in [19]. Additionally, in [14] the authors present numerical examples which show that the connectivity principle is fulfilled, and compute a bifurcation diagram for stationary solutions of (1.4). This supports the claim that fourth-order methods are superior to second-order methods with respect to a smooth continuation of the image contents into the missing domain.

The idea to apply convexity splitting in order to solve (1.4) numerically was born in [13]. The numerical results presented there illustrate the usefulness of this scheme. Although the authors do not analyze the scheme rigorously, based on their numerical results they conjecture unconditional stability. In the following we shall present this numerical scheme and derive some additional properties based on a rigorous analysis of the latter.

The original Cahn-Hilliard equation is a gradient flow in $H^{-1}$ for the energy

$$
E_{1}(u)=\int_{\Omega} \frac{\epsilon}{2}|\nabla u|^{2}+\frac{1}{\epsilon} F(u) d x,
$$

while the fitting term in (1.4) can be derived from a gradient flow in $L^{2}$ for the energy

$$
E_{2}(u)=\frac{1}{2} \int_{\Omega} \lambda(f-u)^{2} d x .
$$

However, note that Equation (1.4) as a whole is no longer a gradient system. Hence, for the discretization in time, we apply the convexity splitting discussed in Section 2 to both functionals $E_{1}$ and $E_{2}$ separately. Namely, we split $E_{1}$ as $E_{1}=E_{1 c}-E_{1 e}$, with

$$
E_{1 c}(u)=\int_{\Omega} \frac{\epsilon}{2}|\nabla u|^{2}+\frac{C_{1}}{2}|u|^{2} d x, \quad E_{1 e}(u)=\int_{\Omega}-\frac{1}{\epsilon} F(u)+\frac{C_{1}}{2}|u|^{2} d x .
$$

A possible splitting for $E_{2}$ is $E_{2}=E_{2 c}-E_{2 e}$ with

$$
E_{2 c}(u)=\frac{1}{2} \int_{\Omega} C_{2}|u|^{2} d x, \quad E_{2 e}(u)=\frac{1}{2} \int_{\Omega}-\lambda(f-u)^{2}+C_{2}|u|^{2} d x .
$$

To make sure that $E_{1 c}, E_{1 e}$ and $E_{2 c}, E_{2 e}$ are strictly convex, the constants $C_{1}$ and $C_{2}$ have to be chosen such that $C_{1}>\frac{1}{\epsilon}, C_{2}>\lambda_{0}$; see [14]. 
Then the resulting discrete time-stepping scheme for an initial condition $U_{0}=u_{0}$ is given by

$$
\frac{U_{k+1}-U_{k}}{\Delta t}=-\nabla_{H^{-1}}\left(E_{1 c}\left(U_{k+1}\right)-E_{1 e}\left(U_{k}\right)\right)-\nabla_{L^{2}}\left(E_{2 c}\left(U_{k+1}\right)-E_{2 e}\left(U_{k}\right)\right),
$$

where $\nabla_{H^{-1}}$ and $\nabla_{L^{2}}$ represent gradient descent with respect to the $H^{-1}$ inner product and the $L^{2}$ inner product respectively. This translates to a numerical scheme of the form

$$
\begin{aligned}
& \frac{U_{k+1}-U_{k}}{\Delta t}+\epsilon \Delta \Delta U_{k+1}-C_{1} \Delta U_{k+1}+C_{2} U_{k+1} \\
= & \frac{1}{\epsilon} \Delta F^{\prime}\left(U_{k}\right)-C_{1} \Delta U_{k}+\lambda\left(f-U_{k}\right)+C_{2} U_{k}, \quad \text { in } \Omega .
\end{aligned}
$$

We enforce Neumann boundary conditions on $\partial \Omega$, i.e.,

$$
\nabla U_{k+1} \cdot \vec{n}=\nabla \Delta U_{k+1} \cdot \vec{n}=0, \quad \text { on } \partial \Omega,
$$

where $\vec{n}$ is the outward pointing normal on $\partial \Omega$, and compute $U_{k+1}$ in (3.2) in the spectral domain using the discrete cosine transform (DCT). The idea to use spectral methods for equations involving Laplacian operators is classical and is based on the fact that the Laplace matrix is diagonalized in the spectral domain. Hence, solving these equations in the spectral domain can be done much faster since matrix multiplication is replaced by scalar multiplication (multiplying with the elements in the main diagonal). Since additionally there also exist fast numerical methods to compute the discrete Fourier/Cosine transform (such as the fast Fourier transform (FFT)) this method has an overall computational advantage. Let $\hat{U}$ be the DCT of $U$ with eigenvalues $\lambda_{i}$. Then Equation (3.2) in $\hat{U}$ reads

$$
\begin{aligned}
& \hat{U}_{k+1}(i, j) \\
= & \frac{\left.\left(1-C_{1} \Delta t\left(\frac{1}{\Delta x^{2}} \lambda_{i}+\frac{1}{\Delta y^{2}} \lambda_{j}\right)+C_{2} \Delta t\right) \hat{U}_{k}(i, j)+\frac{\Delta t}{\epsilon} \Delta \widehat{F^{\prime}\left(U_{k}\right.}\right)(i, j)+\Delta t \lambda\left(\widehat{f-U_{k}}\right)}{1+C_{2} \Delta t+\epsilon \Delta t\left(\frac{1}{\Delta x^{2}} \lambda_{i}+\frac{1}{\Delta y^{2}} \lambda_{j}\right)^{2}-C_{1} \Delta t\left(\frac{1}{\Delta x^{2}} \lambda_{i}+\frac{1}{\Delta y^{2}} \lambda_{j}\right)} .
\end{aligned}
$$

3.1. Rigorous Estimates for the Scheme. From Theorem 2.3 we know that (at least in the spatially discrete framework) the convexity splitting scheme (2.2)(2.4) is unconditionally stable, i.e., separate numerical schemes for the gradient flows of the energies $E_{1}(u)$ and $E_{2}(u)$ are non-increasing for all $\Delta t>0$. But this does not guarantee that the numerical scheme (3.2) is unconditionally stable, since it combines the flows of two energies. In this section we shall analyze the scheme in more detail and derive some rigorous estimates for its solutions. In particular we show that the scheme (3.2) is unconditionally stable in the sense of Definition 2.2. Our results are summarized in the following theorem.

THEOREM 3.1. Let $u$ be the exact solution of (1.4) and $u_{k}=u(k \Delta t)$ the exact solution at time $k \Delta t$, for a time step $\Delta t>0$ and $k \in \mathbb{N}$. Let $U_{k}$ be the $k$ th iterate of (3.2) with constants $C_{1}>1 / \epsilon, C_{2}>\lambda_{0}$. Then the following statements are true:

(i) Under the assumption that $\left\|u_{t t}\right\|_{-1}$ and $\left\|\nabla \Delta u_{t}\right\|_{2}$ are bounded, the numerical scheme (3.2) is consistent with the continuous Equation (1.4) and of order one in time. 
Under the additional assumption that

$$
F^{\prime \prime}\left(U_{k-1}\right) \leq K
$$

for a nonnegative constant $K$, we further have

(ii) The solution sequence $U_{k}$ is bounded on a finite time interval $[0, T]$, for all $\Delta t>0$. In particular for $k \Delta t \leq T, T>0$ fixed, we have for every $\Delta t>0$

$$
\begin{aligned}
& \left\|\nabla U_{k}\right\|_{2}^{2}+\Delta t K_{1}\left\|\Delta U_{k}\right\|_{2}^{2} \\
\leq & e^{K_{2} T}\left(\left\|\nabla U_{0}\right\|_{2}^{2}+\Delta t K_{1}\left\|\Delta U_{0}\right\|_{2}^{2}+\Delta t T C\left(\Omega, D, \lambda_{0}, f\right)\right),
\end{aligned}
$$

for suitable constants $K_{1}$ and $K_{2}$, and constant $C$ depending on $\Omega, D, \lambda_{0}, f$ only.

(iii) The discretization error $e_{k}$, given by $e_{k}=u_{k}-U_{k}$, converges to zero as $\Delta t \rightarrow 0$. In particular, we have for $k \Delta t \leq T, T>0$ fixed, that

$$
\left\|\nabla e_{k}\right\|_{2}^{2}+\Delta t \frac{C_{1}}{\tilde{C}}\left\|\Delta e_{k}\right\|_{2}^{2} \leq \frac{T}{\tilde{C}} e^{K_{1} T} \cdot C \cdot(\Delta t)^{2},
$$

for suitable constants $C, \tilde{C}, K_{1}$.

REMARK 3.1. Note that our assumptions for the consistency of the numerical scheme only hold if the time derivative of the solution of the continuous Equation (1.4) is uniformly bounded. This is true for smooth and bounded solutions of the equation.

Further, since we are interested in bounded solutions $U_{k}$ of the discrete Equation (3.2), it is natural to assume (3.4), i.e., that the nonlinearity $F^{\prime \prime}$ in the previous time step $(k-1) \Delta t$ is bounded. Also note that the constant $K$ in (3.4) can be chosen arbitrarily large.

The proof of Theorem 3.1 is organized in Propositions 3.2-3.4.

Proposition 3.2 (Consistency (i)). Under the same assumptions as in Theorem 3.1, and in particular under the assumption that $\left\|u_{t t}\right\|_{-1}$ and $\left\|\nabla \Delta u_{t}\right\|_{2}$ are bounded, the numerical scheme (3.2) is consistent with the continuous Equation (1.4) with $\left\|\tau_{k}\right\|_{-1}=O(\Delta t)$ as $\Delta t \rightarrow 0$, where $\tau_{k}$ is the local truncation error as defined in Equation (2.6) above.

Proof. Let $\tau_{k}$ be the local truncation error defined as in (2.6). Then

$$
\tau_{k}=\tau_{k}^{1}+\tau_{k}^{2}
$$

with

$$
\begin{aligned}
\tau_{k}^{1} & =\frac{u_{k+1}-u_{k}}{\Delta t}-u_{t}(k \Delta t) \\
\tau_{k}^{2} & =\epsilon \Delta \Delta\left(u_{k+1}-u_{k}\right)-C_{1} \Delta\left(u_{k+1}-u_{k}\right)+C_{2}\left(u_{k+1}-u_{k}\right) \\
& =\epsilon \Delta t \Delta^{2} \frac{u_{k+1}-u_{k}}{\Delta t}-C_{1} \Delta t \Delta \frac{u_{k+1}-u_{k}}{\Delta t}+C_{2} \Delta t \frac{u_{k+1}-u_{k}}{\Delta t},
\end{aligned}
$$

i.e.,

$\tau_{k}=\frac{u_{k+1}-u_{k}}{\Delta t}+\epsilon \Delta^{2} u_{k+1}-\frac{1}{\epsilon} \Delta F^{\prime}\left(u_{k}\right)-\lambda\left(f-u_{k}\right)-C_{1} \Delta\left(u_{k+1}-u_{k}\right)+C_{2}\left(u_{k+1}-u_{k}\right)$. 
Using standard Taylor series arguments and assuming that $\left\|u_{t t}\right\|_{-1}$ and $\left\|\nabla \Delta u_{t}\right\|_{2}$ are bounded we deduce that the global truncation error $\tau$ is given by

$$
\tau=\max _{k}\left\|\tau_{k}\right\|_{-1}=O(\Delta t) \quad \text { as } \Delta t \rightarrow 0 .
$$

Proposition 3.3 (Unconditional stability (ii)). Under the same assumptions as in Theorem 3.1 and in particular assuming that (3.4) holds, the solution sequence $U_{k}$ fulfills (3.5). This gives boundedness of the solution sequence on $[0, T]$.

Proof. We consider our discrete model

$$
\begin{aligned}
& \frac{U_{k+1}-U_{k}}{\Delta t}+\epsilon \Delta \Delta U_{k+1}-C_{1} \Delta U_{k+1}+C_{2} U_{k+1} \\
= & \frac{1}{\epsilon} \Delta F^{\prime}\left(U_{k}\right)-C_{1} \Delta U_{k}+\lambda\left(f-U_{k}\right)+C_{2} U_{k},
\end{aligned}
$$

multiply the equation with $-\Delta U_{k+1}$ and integrate over $\Omega$. We obtain

$$
\begin{aligned}
& \frac{1}{\Delta t}\left(\left\|\nabla U_{k+1}\right\|_{2}^{2}-\left\langle\nabla U_{k}, \nabla U_{k+1}\right\rangle_{2}\right)+\epsilon\left\|\nabla \Delta U_{k+1}\right\|_{2}^{2}+C_{1}\left\|\Delta U_{k+1}\right\|_{2}^{2}+C_{2}\left\|\nabla U_{k+1}\right\|_{2}^{2} \\
& =\frac{1}{\epsilon}\left\langle F^{\prime \prime}\left(U_{k}\right) \nabla U_{k}, \nabla \Delta U_{k+1}\right\rangle_{2}+C_{1}\left\langle\Delta U_{k}, \Delta U_{k+1}\right\rangle_{2} \\
& +\left\langle\nabla \lambda\left(f-U_{k}\right), \nabla U_{k+1}\right\rangle_{2}+C_{2}\left\langle\nabla U_{k}, \nabla U_{k+1}\right\rangle_{2} .
\end{aligned}
$$

Using Young's inequality we obtain

$$
\begin{gathered}
\frac{1}{2 \Delta t}\left(\left\|\nabla U_{k+1}\right\|_{2}^{2}-\left\|\nabla U_{k}\right\|_{2}^{2}\right)+\epsilon\left\|\nabla \Delta U_{k+1}\right\|_{2}^{2}+C_{1}\left\|\Delta U_{k+1}\right\|_{2}^{2}+C_{2}\left\|\nabla U_{k+1}\right\|_{2}^{2} \\
\leq \frac{1}{2 \epsilon \delta}\left\|F^{\prime \prime}\left(U_{k}\right) \nabla U_{k}\right\|_{2}^{2}+\frac{\delta}{2 \epsilon}\left\|\nabla \Delta U_{k+1}\right\|_{2}^{2}+\frac{C_{1}}{2}\left\|\Delta U_{k}\right\|_{2}^{2}+\frac{C_{1}}{2}\left\|\Delta U_{k+1}\right\|_{2}^{2} \\
+\frac{C_{2}}{2}\left\|\nabla U_{k}\right\|_{2}^{2}+\frac{C_{2}}{2}\left\|\nabla U_{k+1}\right\|_{2}^{2}+\frac{1}{2}\left\|\nabla \lambda\left(f-U_{k}\right)\right\|_{2}^{2}+\frac{1}{2}\left\|\nabla U_{k+1}\right\|_{2}^{2} .
\end{gathered}
$$

Using the estimate

$$
\left\|\nabla \lambda\left(f-U_{k}\right)\right\|_{2}^{2} \leq 2 \lambda_{0}^{2}\left\|\nabla U_{k}\right\|_{2}^{2}+C\left(\Omega, D, \lambda_{0}, f\right)
$$

and reordering the terms, we obtain

$$
\begin{aligned}
& \left(\frac{1}{2 \Delta t}+\frac{C_{2}}{2}-\frac{1}{2}\right)\left\|\nabla U_{k+1}\right\|_{2}^{2}+\frac{C_{1}}{2}\left\|\Delta U_{k+1}\right\|_{2}^{2}+\left(\epsilon-\frac{\delta}{2 \epsilon}\right)\left\|\nabla \Delta U_{k+1}\right\|_{2}^{2} \\
\leq & \left(\frac{1}{2 \Delta t}+\frac{C_{2}}{2}+\lambda_{0}^{2}\right)\left\|\nabla U_{k}\right\|_{2}^{2}+\frac{1}{2 \epsilon \delta}\left\|F^{\prime \prime}\left(U_{k}\right) \nabla U_{k}\right\|_{2}^{2}+\frac{C_{1}}{2}\left\|\Delta U_{k}\right\|_{2}^{2}+C\left(\Omega, D, \lambda_{0}, f\right) .
\end{aligned}
$$

By choosing $\delta=2 \epsilon^{2}$, the third term on the left side of the inequality is zero. Because of Assumption (3.4) we obtain the following bound on the right side of the inequality

$$
\left\|F^{\prime \prime}\left(U_{k}\right) \nabla U_{k}\right\|_{2}^{2} \leq K^{2}\left\|\nabla U_{k}\right\|_{2}^{2}
$$

and we have

$$
\begin{aligned}
& \left(\frac{1}{2 \Delta t}+\frac{C_{2}}{2}-\frac{1}{2}\right)\left\|\nabla U_{k+1}\right\|_{2}^{2}+\frac{C_{1}}{2}\left\|\Delta U_{k+1}\right\|_{2}^{2} \\
\leq & \left(\frac{1}{2 \Delta t}+\frac{C_{2}}{2}+\lambda_{0}^{2}+\frac{K^{2}}{4 \epsilon^{3}}\right)\left\|\nabla U_{k}\right\|_{2}^{2}+\frac{C_{1}}{2}\left\|\Delta U_{k}\right\|_{2}^{2}+C\left(\Omega, D, \lambda_{0}, f\right) .
\end{aligned}
$$


Now we multiply the above inequality by $2 \Delta t$ and define

$$
\begin{aligned}
& \tilde{C}=1+\Delta t\left(C_{2}-1\right), \\
& \tilde{C}=1+\Delta t\left(C_{2}+2 \lambda_{0}^{2}+\frac{K^{2}}{2 \epsilon^{3}}\right) .
\end{aligned}
$$

Since $C_{2}$ is chosen greater than $\lambda_{0}>1$, the first coefficient $\tilde{C}$ is positive and we can divide the inequality by it. We obtain

$$
\left\|\nabla U_{k+1}\right\|_{2}^{2}+\Delta t \frac{C_{1}}{\tilde{C}}\left\|\Delta U_{k+1}\right\|_{2}^{2} \leq \frac{\tilde{\tilde{C}}}{\tilde{C}}\left\|\nabla U_{k}\right\|_{2}^{2}+\Delta t \frac{C_{1}}{\tilde{C}}\left\|\Delta U_{k}\right\|_{2}^{2}+\Delta t C\left(\Omega, D, \lambda_{0}, f\right),
$$

where we updated the constant $C\left(\Omega, D, \lambda_{0}, f\right)$ by $C\left(\Omega, D, \lambda_{0}, f\right) / \tilde{C}$.

Since $\frac{\tilde{C}}{\tilde{C}} \geq 1$, we can multiply the second term on the right side of the inequality by this quotient to obtain

$$
\left\|\nabla U_{k+1}\right\|_{2}^{2}+\Delta t \frac{C_{1}}{\tilde{C}}\left\|\Delta U_{k+1}\right\|_{2}^{2} \leq \frac{\tilde{\tilde{C}}}{\tilde{C}}\left(\left\|\nabla U_{k}\right\|_{2}^{2}+\Delta t \frac{C_{1}}{\tilde{C}}\left\|\Delta U_{k}\right\|_{2}^{2}\right)+\Delta t C\left(\Omega, D, \lambda_{0}, f\right) .
$$

We deduce by induction that

$$
\begin{gathered}
\left\|\nabla U_{k}\right\|_{2}^{2}+\Delta t \frac{C_{1}}{\tilde{C}}\left\|\Delta U_{k}\right\|_{2}^{2} \leq\left(\frac{\tilde{\tilde{C}}}{\tilde{C}}\right)^{k}\left(\left\|\nabla U_{0}\right\|_{2}^{2}+\Delta t \frac{C_{1}}{\tilde{C}}\left\|\Delta U_{0}\right\|_{2}^{2}\right) \\
+\Delta t \sum_{i=0}^{k-1}\left(\frac{\tilde{\tilde{C}}}{\tilde{C}}\right)^{i} C\left(\Omega, D, \lambda_{0}, f\right) \\
=\frac{\left(1+K_{2} \Delta t\right)^{k}}{\left(1+K_{1} \Delta t\right)^{k}}\left(\left\|\nabla U_{0}\right\|_{2}^{2}+\Delta t \frac{C_{1}}{\tilde{C}}\left\|\Delta U_{0}\right\|_{2}^{2}\right) \\
+\Delta t \sum_{i=0}^{k-1} \frac{\left(1+K_{2} \Delta t\right)^{i}}{\left(1+K_{1} \Delta t\right)^{i}} C\left(\Omega, D, \lambda_{0}, f\right) .
\end{gathered}
$$

For $k \Delta t \leq T$ we have

$$
\begin{gathered}
\left\|\nabla U_{k}\right\|_{2}^{2}+\Delta t \frac{C_{1}}{\tilde{C}}\left\|\Delta U_{k}\right\|_{2}^{2} \leq e^{\left(K_{2}-K_{1}\right) T}\left(\left\|\nabla U_{0}\right\|_{2}^{2}+\Delta t \frac{C_{1}}{\tilde{C}}\left\|\Delta U_{0}\right\|_{2}^{2}\right) \\
+\Delta t T e^{\left(K_{2}-K_{1}\right) T} C\left(\Omega, D, \lambda_{0}, f\right) \\
=e^{\left(K_{2}-K_{1}\right) T}\left(\left\|\nabla U_{0}\right\|_{2}^{2}+\Delta t \frac{C_{1}}{\tilde{C}}\left\|\Delta U_{0}\right\|_{2}^{2}\right. \\
\left.+\Delta t T C\left(\Omega, D, \lambda_{0}, f\right)\right)
\end{gathered}
$$

which gives boundedness of the solution sequence on $[0, T]$ for any $T>0$, assuming that (3.4) holds.

The convergence of the discrete solution to the continuous one as the time step $\Delta t \rightarrow 0$ is verified in the following proposition.

Proposition 3.4 (Convergence (iii)). Under the same assumptions as in Theorem 3.1 and in particular under Assumption (3.4) the discretization error $e_{k}$ fulfills (3.6). 
In order to prove Proposition 3.4 we need the following auxiliary lemma.

LEMMA 3.2. The error $e_{k}$ between the exact and approximate solution defined as in Theorem 3.1 fulfills

$$
\int_{\Omega} e_{k} d x=O\left((\Delta t)^{2}\right)
$$

Proof. [Proof of Lemma 3.2] Because of the fidelity term in (1.4) and (3.2), solutions of these equations are not mass preserving, i.e., $\int_{\Omega} e_{k}$ does not in general vanish. In fact we have, for a solution $u_{k}$ of (1.4),

$$
\begin{aligned}
\frac{d}{d t} \int_{\Omega} u_{k} & =-\epsilon \int_{\Omega} \Delta^{2} u_{k}+\frac{1}{\epsilon} \int_{\Omega} \Delta F^{\prime}\left(u_{k}\right)+\int_{\Omega} \lambda\left(f-u_{k}\right) \\
& =-\epsilon \int_{\partial \Omega} \nabla \Delta u_{k} \cdot \vec{n}+\frac{1}{\epsilon} \int_{\partial \Omega} \nabla F^{\prime}\left(u_{k}\right) \cdot \vec{n}+\int_{\Omega} \lambda\left(f-u_{k}\right),
\end{aligned}
$$

where we have used Gauss divergence theorem to obtain the boundary integrals. Assuming zero Neumann boundary conditions as in (3.3) the two boundary integrals vanish, and hence

$$
\frac{d}{d t} \int_{\Omega} u_{k}=\int_{\Omega} \lambda\left(f-u_{k}\right) .
$$

In particular

$$
\frac{d}{d t} \int_{D} u_{k}=0
$$

A similar computation for the discrete solution of (3.2) shows that

$$
\left(\frac{1}{\Delta t}+C_{2}\right) \int_{\Omega}\left(U_{k+1}-U_{k}\right)=\int_{\Omega} \lambda\left(f-U_{k}\right),
$$

and in particular

$$
\left(\frac{1}{\Delta t}+C_{2}\right) \int_{D}\left(U_{k+1}-U_{k}\right)=0 .
$$

Next, let us follow the lines of the consistency proof in (3.7). Then the discretization error $e_{k}$ satisfies

$$
\begin{aligned}
& \frac{e_{k+1}-e_{k}}{\Delta t}+\epsilon \Delta^{2} e_{k+1}-C_{1} \Delta e_{k+1}+C_{2} e_{k+1} \\
= & \frac{1}{\Delta t}\left(u_{k+1}-u_{k}\right)-\frac{1}{\Delta t}\left(U_{k+1}-U_{k}\right)+\epsilon \Delta^{2} u_{k+1}-\epsilon \Delta^{2} U_{k+1} \\
& \quad-C_{1} \Delta u_{k+1}+C_{1} \Delta U_{k+1}+C_{2} u_{k+1}-C_{2} U_{k+1} \\
= & -\left(\frac{1}{\epsilon} \Delta F^{\prime}\left(U_{k}\right)-C_{1} \Delta U_{k}+\lambda\left(f-U_{k}\right)+C_{2} U_{k}\right) \\
& \quad+\left(\frac{1}{\epsilon} \Delta F^{\prime}\left(u_{k}\right)+\lambda\left(f-u_{k}\right)-C_{1} \Delta u_{k}+C_{2} u_{k}\right)+\tau_{k} \\
= & -\left(\frac{1}{\epsilon} \Delta\left(F^{\prime}\left(U_{k}\right)-F^{\prime}\left(u_{k}\right)\right)-C_{1} \Delta\left(U_{k}-u_{k}\right)+C_{2}\left(U_{k}-u_{k}\right)-\lambda\left(U_{k}-u_{k}\right)\right)+\tau_{k} .
\end{aligned}
$$


As before, integrating over $\Omega$, applying Gauss divergence theorem and the zero Neumann boundary conditions for $u_{k}$ and $U_{k}$, we get

$$
\left(\frac{1}{\Delta t}+C_{2}\right) \int_{\Omega}\left(e_{k+1}-e_{k}\right)+\int_{\Omega} \lambda e_{k}=\int \tau_{k}
$$

where

$$
\begin{aligned}
\int_{\Omega} \tau_{k} & =\left(\frac{1}{\Delta t}+C_{2}\right) \int_{\Omega}\left(u_{k+1}-u_{k}\right)-\int_{\Omega}\left(u_{k}\right)_{t} \\
& =\left(\frac{1}{\Delta t}+C_{2}\right) \int_{\Omega}\left(u_{k}+\Delta t\left(u_{k}\right)_{t}+O\left((\Delta t)^{2}\right)-u_{k}\right)-\int_{\Omega}\left(u_{k}\right)_{t} \\
& =O(\Delta t) .
\end{aligned}
$$

Now, to prove our claim we apply induction on $k$. First, assuming that $u_{0}=U_{0}$ in $\Omega$, we have that

$$
\int_{\Omega} e_{0}=0
$$

and hence

$$
\left(\frac{1}{\Delta t}+C_{2}\right) \int_{\Omega} e_{1}=O(\Delta t)
$$

Assuming that assertion (3.12) holds for all indices $\leq k$ and using (3.9) and (3.10) we have, for (3.11),

$$
\begin{aligned}
\left(\frac{1}{\Delta t}+C_{2}\right) \int_{\Omega}\left(e_{k+1}-e_{k}\right)+\int_{\Omega} \lambda e_{k} & =\int \tau_{k} \\
\left(\frac{1}{\Delta t}+C_{2}\right) \int_{\Omega} e_{k+1}-O(\Delta t)+\lambda_{0}\left(\frac{1}{\Delta t}+C_{2}\right)^{-1} O(\Delta t) & =O(\Delta t) \\
\left(\frac{1}{\Delta t}+C_{2}\right) \int_{\Omega} e_{k+1} & =O(\Delta t),
\end{aligned}
$$

and hence

$$
\left(1+C_{2} \Delta t\right) \int_{\Omega} e_{k}=O\left((\Delta t)^{2}\right)
$$

for all $k \geq 0$.

We continue with the proof of Proposition 3.4.

Proof. [Proof of Proposition 3.4] In the proof of Lemma 3.2 we have used the consistency result (3.7) to show that the discretization error $e_{k}$ satisfies

$$
\begin{aligned}
& \frac{e_{k+1}-e_{k}}{\Delta t}+\epsilon \Delta^{2} e_{k+1}-C_{1} \Delta e_{k+1}+C_{2} e_{k+1} \\
= & -\left(\frac{1}{\epsilon} \Delta\left(F^{\prime}\left(U_{k}\right)-F^{\prime}\left(u_{k}\right)\right)-C_{1} \Delta\left(U_{k}-u_{k}\right)+C_{2}\left(U_{k}-u_{k}\right)-\lambda\left(U_{k}-u_{k}\right)\right)+\tau_{k} .
\end{aligned}
$$


Multiplication with $-\Delta e_{k+1}$ leads to

$$
\begin{aligned}
& \frac{1}{\Delta t}\left\langle\nabla\left(e_{k+1}-e_{k}\right), \nabla e_{k+1}\right\rangle_{2}+\epsilon\left\|\nabla \Delta e_{k+1}\right\|_{2}^{2}+C_{1}\left\|\Delta e_{k+1}\right\|_{2}^{2}+C_{2}\left\|\nabla e_{k+1}\right\|_{2}^{2} \\
=\frac{1}{\epsilon}\langle\Delta & \left.\left(F^{\prime}\left(U_{k}\right)-F^{\prime}\left(u_{k}\right)\right), \Delta e_{k+1}\right\rangle_{2}-C_{1}\left\langle\Delta\left(U_{k}-u_{k}\right), \Delta e_{k+1}\right\rangle_{2} \\
& \quad+\left\langle\nabla \lambda\left(U_{k}-u_{k}\right), \nabla e_{k+1}\right\rangle_{2}-C_{2}\left\langle\nabla\left(U_{k}-u_{k}\right), \nabla e_{k+1}\right\rangle_{2}+\left\langle\nabla \Delta^{-1} \tau_{k}, \nabla \Delta e_{k+1}\right\rangle_{2} .
\end{aligned}
$$

Further, because

$$
\frac{1}{\Delta t}\left(\left\|\nabla e_{k+1}\right\|_{2}^{2}-\left\langle\nabla e_{k}, \nabla e_{k+1}\right\rangle_{2}\right) \geq \frac{1}{2 \Delta t}\left(\left\|\nabla e_{k+1}\right\|_{2}^{2}-\left\|\nabla e_{k}\right\|_{2}^{2}\right),
$$

we obtain

$$
\begin{aligned}
& \frac{1}{2 \Delta t}\left(\left\|\nabla e_{k+1}\right\|_{2}^{2}-\left\|\nabla e_{k}\right\|_{2}^{2}\right)+\epsilon\left\|\nabla \Delta e_{k+1}\right\|_{2}^{2}+C_{1}\left\|\Delta e_{k+1}\right\|_{2}^{2}+C_{2}\left\|\nabla e_{k+1}\right\|_{2}^{2} \\
\leq & \frac{1}{\epsilon}\left\langle\Delta\left(F^{\prime}\left(U_{k}\right)-F^{\prime}\left(u_{k}\right)\right), \Delta e_{k+1}\right\rangle_{2}+C_{1}\left\langle\Delta e_{k}, \Delta e_{k+1}\right\rangle_{2}-\left\langle\nabla \lambda e_{k}, \nabla e_{k+1}\right\rangle_{2} \\
& +C_{2}\left\langle\nabla e_{k}, \nabla e_{k+1}\right\rangle_{2}+\left\langle\nabla \Delta^{-1} \tau_{k}, \nabla \Delta e_{k+1}\right\rangle_{2} .
\end{aligned}
$$

Applying Young's inequality leads to

$$
\begin{gathered}
\frac{1}{2 \Delta t}\left(\left\|\nabla e_{k+1}\right\|_{2}^{2}-\left\|\nabla e_{k}\right\|_{2}^{2}\right)+\epsilon\left\|\nabla \Delta e_{k+1}\right\|_{2}^{2}+C_{1}\left\|\Delta e_{k+1}\right\|_{2}^{2}+C_{2}\left\|\nabla e_{k+1}\right\|_{2}^{2} \\
\leq-\frac{1}{\epsilon}\left\langle\left(F^{\prime \prime}\left(U_{k}\right) \nabla U_{k}-F^{\prime \prime}\left(u_{k}\right) \nabla u_{k}\right), \nabla \Delta e_{k+1}\right\rangle_{2}+\frac{C_{1}}{2 \delta_{1}}\left\|\Delta e_{k}\right\|_{2}^{2}+\frac{C_{1} \delta_{1}}{2}\left\|\Delta e_{k+1}\right\|_{2}^{2} \\
+\frac{\lambda_{0}^{2}}{2 \delta_{3}}\left\|\nabla e_{k}\right\|_{2}^{2}+\frac{\delta_{3}}{2}\left\|\nabla e_{k+1}\right\|_{2}^{2}+\frac{C_{2}}{2 \delta_{2}}\left\|\nabla e_{k}\right\|_{2}^{2}+\frac{C_{2} \delta_{2}}{2}\left\|\nabla e_{k+1}\right\|_{2}^{2} \\
+\frac{1}{2 \delta_{4}}\left\|\tau_{k}\right\|_{-1}^{2}+\frac{\delta_{4}}{2}\left\|\nabla \Delta e_{k+1}\right\|_{2}^{2} .
\end{gathered}
$$

Let us consider the remaining inner product in the last inequality:

$$
\begin{aligned}
& -\frac{1}{\epsilon}\left\langle\left(F^{\prime \prime}\left(U_{k}\right) \nabla U_{k}-F^{\prime \prime}\left(u_{k}\right) \nabla u_{k}\right), \nabla \Delta e_{k+1}\right\rangle_{2} \\
= & \frac{1}{\epsilon}\left\langle F^{\prime \prime}\left(U_{k}\right) \nabla e_{k}, \nabla \Delta e_{k+1}\right\rangle_{2}+\frac{1}{\epsilon}\left\langle\left(F^{\prime \prime}\left(u_{k}\right)-F^{\prime \prime}\left(U_{k}\right)\right) \nabla u_{k}, \nabla \Delta e_{k+1}\right\rangle_{2} \\
\leq & \frac{1}{2 \delta_{5} \epsilon}\left\|F^{\prime \prime}\left(U_{k}\right)\left|\nabla e_{k}\right|\right\|_{2}^{2}+\frac{1}{2 \delta_{6} \epsilon}\left\|\left(F^{\prime \prime}\left(u_{k}\right)-F^{\prime \prime}\left(U_{k}\right)\right)\left|\nabla u_{k}\right|\right\|_{2}^{2}+\left(\frac{\delta_{5}}{2 \epsilon}+\frac{\delta_{6}}{2 \epsilon}\right)\left\|\nabla \Delta e_{k+1}\right\|_{2}^{2} .
\end{aligned}
$$

Next we assume that (3.4) holds and that $\nabla u_{k}$ is uniformly bounded on $[0, T]-$ in particular, that

$$
\exists \tilde{\tilde{K}}>0 \text { such that }\left\|\nabla u_{k}\right\|_{2} \leq \tilde{\tilde{K}} \text { for all } k \Delta t<T .
$$

The latter assumption will be proven in Lemma 3.3 just after the end of this proof. Moreover, since $F^{\prime \prime}$ is locally Lipschitz continuous we obtain

$$
\begin{aligned}
& -\frac{1}{\epsilon}\left\langle\left(F^{\prime \prime}\left(U_{k}\right) \nabla U_{k}-F^{\prime \prime}\left(u_{k}\right) \nabla u_{k}\right), \nabla \Delta e_{k+1}\right\rangle_{2} \\
\leq & \frac{C}{2 \delta_{5} \epsilon}\left\|\nabla e_{k}\right\|_{2}^{2}+\frac{C}{2 \delta_{6} \epsilon}\left\|e_{k}\right\|_{2}^{2}+\left(\frac{\delta_{5}}{2 \epsilon}+\frac{\delta_{6}}{2 \epsilon}\right)\left\|\nabla \Delta e_{k+1}\right\|_{2}^{2},
\end{aligned}
$$


where we have set $C$ to be a universal constant for all bounds. Further, using Lemma 3.2 and

$$
\begin{aligned}
\left\|e_{k}\right\|_{2}^{2} & =\left\|e_{k}-O(\Delta t)^{2}+O(\Delta t)^{2}\right\|_{2}^{2} \\
& \leq 2\left\|e_{k}-O(\Delta t)^{2}\right\|_{2}^{2}+2\left\|O(\Delta t)^{2}\right\|_{2}^{2},
\end{aligned}
$$

we can apply the Poincaré inequality to the $L^{2}$ norm of $e_{k}$. In sum we get

$$
\begin{gathered}
\left(\frac{1}{2 \Delta t}+C_{2}\left(1-\frac{\delta_{2}}{2}\right)-\frac{\delta_{3}}{2}\right)\left\|\nabla e_{k+1}\right\|_{2}^{2}+C_{1}\left(1-\frac{\delta_{1}}{2}\right)\left\|\Delta e_{k+1}\right\|_{2}^{2} \\
+\left(\epsilon-\frac{\delta_{4}}{2}-\frac{\delta_{5}+\delta_{6}}{2 \epsilon}\right)\left\|\nabla \Delta e_{k+1}\right\|_{2}^{2} \\
\leq\left(\frac{1}{2 \Delta t}+\frac{\lambda_{0}^{2}}{2 \delta_{3}}+\frac{C_{2}}{2 \delta_{2}}+\frac{C}{2 \delta_{5} \epsilon}+\frac{C}{\delta_{6} \epsilon}\right)\left\|\nabla e_{k}\right\|_{2}^{2}+\frac{C_{1}}{2 \delta_{1}}\left\|\Delta e_{k}\right\|_{2}^{2} \\
+\frac{1}{2 \delta_{4}}\left\|\tau_{k}\right\|_{-1}^{2}+\frac{C}{\epsilon \delta_{6}}\left\|O(\Delta t)^{2}\right\|_{2}^{2} .
\end{gathered}
$$

Next we choose $\delta_{1}=1$ and multiply the inequality with $2 \Delta t$ :

$$
\begin{gathered}
\left(1+\Delta t\left(C_{2}\left(2-\delta_{2}\right)-\delta_{3}\right)\right)\left\|\nabla e_{k+1}\right\|_{2}^{2}+\Delta t C_{1}\left\|\Delta e_{k+1}\right\|_{2}^{2} \\
+\Delta t\left(2 \epsilon-\delta_{4}-\frac{\delta_{5}+\delta_{6}}{\epsilon}\right)\left\|\nabla \Delta e_{k+1}\right\|_{2}^{2} \\
\leq\left(1+\Delta t\left(\frac{\lambda_{0}^{2}}{\delta_{3}}+\frac{C_{2}}{\delta_{2}}+\frac{C}{\delta_{5} \epsilon}+\frac{2 C}{\delta_{6} \epsilon}\right)\right)\left\|\nabla e_{k}\right\|_{2}^{2}+\Delta t C_{1}\left\|\Delta e_{k}\right\|_{2}^{2} \\
+\frac{\Delta t}{\delta_{4}}\left\|\tau_{k}\right\|_{-1}^{2}+\Delta t \frac{2 C}{\epsilon \delta_{6}}\left\|O(\Delta t)^{2}\right\|_{2}^{2} .
\end{gathered}
$$

Let

$$
\tilde{C}=1+\Delta t\left(C_{2}\left(2-\delta_{2}\right)-\delta_{3}\right), \quad \tilde{\tilde{C}}=1+\Delta t\left(\frac{\lambda_{0}^{2}}{\delta_{3}}+\frac{C_{2}}{\delta_{2}}+\frac{C}{\delta_{5} \epsilon}+\frac{2 C}{\delta_{6} \epsilon}\right) .
$$

Now, by choosing all $\delta$ s such that the coefficients of all terms in the inequality are nonnegative and the quotient $\tilde{\tilde{C}} / \tilde{C} \geq 1$, and by estimating the last term on the left side from below by zero, we get

$$
\begin{aligned}
& \left\|\nabla e_{k+1}\right\|_{2}^{2}+\Delta t \frac{C_{1}}{\tilde{C}}\left\|\Delta e_{k+1}\right\|_{2}^{2} \\
\leq & \frac{\tilde{\tilde{C}}}{\tilde{C}}\left\|\nabla e_{k}\right\|_{2}^{2}+\Delta t \frac{C_{1}}{\tilde{C}}\left\|\Delta e_{k}\right\|_{2}^{2}+\frac{\Delta t}{\tilde{C}}\left(\frac{1}{\delta_{4}}\left\|\tau_{k}\right\|_{-1}^{2}+\frac{2 C}{\delta_{6} \epsilon}\left\|O(\Delta t)^{2}\right\|_{2}^{2}\right),
\end{aligned}
$$

and because $\tilde{\tilde{C}} / \tilde{C} \geq 1$ we further have

$$
\begin{aligned}
\left\|\nabla e_{k+1}\right\|_{2}^{2}+\Delta t \frac{C_{1}}{\tilde{C}}\left\|\Delta e_{k+1}\right\|_{2}^{2} \leq \frac{\tilde{\tilde{C}}}{\tilde{C}}\left(\left\|\nabla e_{k}\right\|_{2}^{2}+\right. & \left.\Delta t \frac{C_{1}}{\tilde{C}}\left\|\Delta e_{k}\right\|_{2}^{2}\right) \\
& +\frac{\Delta t}{\tilde{C}}\left(\frac{1}{\delta_{4}}\left\|\tau_{k}\right\|_{-1}^{2}+\frac{2 C}{\delta_{6} \epsilon}\left\|O(\Delta t)^{2}\right\|_{2}^{2}\right) .
\end{aligned}
$$


By induction on $k$ we obtain

$$
\begin{gathered}
\left\|\nabla e_{k+1}\right\|_{2}^{2}+\Delta t \frac{C_{1}}{\tilde{C}}\left\|\Delta e_{k+1}\right\|_{2}^{2} \\
\leq\left(\frac{\tilde{\tilde{C}}}{\tilde{C}}\right)^{k+1}\left(\left\|\nabla e_{0}\right\|_{2}^{2}+\Delta t \frac{C_{1}}{\tilde{C}}\left\|\Delta e_{0}\right\|_{2}^{2}\right) \\
+\frac{\Delta t}{\tilde{C}} \sum_{i=0}^{k}\left(\frac{\tilde{\tilde{C}}}{\tilde{C}}\right)^{i} \cdot\left(\frac{1}{\delta_{4}} \max _{i \leq k}\left\{\left\|\tau_{i}\right\|_{-1}^{2}\right\}+\frac{2 C}{\delta_{6} \epsilon}\|O(\Delta t)\|_{2}^{2}\right) \\
=\frac{\Delta t}{\tilde{C}} \sum_{i=0}^{k}\left(1+K_{1} \Delta t\right)^{i} \cdot\left(\frac{1}{\delta_{4}} \max _{i \leq k}\left\{\left\|\tau_{i}\right\|_{-1}^{2}\right\}+\frac{2 C}{\delta_{6} \epsilon}\left\|O(\Delta t)^{2}\right\|_{2}^{2}\right) \\
\leq \frac{\Delta t}{\tilde{C}} \cdot k \cdot e^{K_{1} k \Delta t} \cdot\left(\frac{1}{\delta_{4}} \max _{i \leq k}\left\{\left\|\tau_{i}\right\|_{-1}^{2}\right\}+\frac{2 C}{\delta_{6} \epsilon}\left\|O(\Delta t)^{2}\right\|_{2}^{2}\right),
\end{gathered}
$$

where we have used the fact that $e_{0}=0$ and $1 \leq \frac{\tilde{\tilde{C}}}{\tilde{C}}=1+K_{1} \Delta t$. Hence, by using the consistency result (3.8) we conclude, for $k \Delta t \leq T$, that

$$
\left\|\nabla e_{k}\right\|_{2}^{2}+\Delta t \frac{C_{1}}{\tilde{C}}\left\|\Delta e_{k}\right\|_{2}^{2} \leq \frac{T}{\tilde{C}} e^{K_{1} T} \cdot C \cdot(\Delta t)^{2} .
$$

From $[13,14]$ we know that the solution $u_{k}$ to the continuous equation globally exists and is uniformly bounded in $L^{2}(\Omega)$. Next we show that assumption (3.13) holds.

Lemma 3.3. Let $u_{k}$ be the exact solution of (1.4) at time $t=k \Delta t$ and let $T>0$. Then there exists a constant $C>0$ such that $\left\|\nabla u_{k}\right\|_{2} \leq C$ for all $k \Delta t<T$.

Proof. Let $K(u)=-\epsilon \Delta u+\frac{1}{\epsilon} F^{\prime}(u)$. We multiply the continuous evolution Equation (1.4) with $K(u)$ and obtain

$$
\left\langle u_{t}, K(u)\right\rangle_{2}=\langle\Delta K(u), K(u)\rangle_{2}+\langle\lambda(f-u), K(u)\rangle_{2} .
$$

Let us further define

$$
E(u):=\frac{\epsilon}{2} \int_{\Omega}|\nabla u|^{2} d x+\frac{1}{\epsilon} \int_{\Omega} F(u) d x .
$$

Then we have

$$
\begin{aligned}
\left\langle u_{t}, K(u)\right\rangle_{2} & =\left\langle u_{t},-\epsilon \Delta u+\frac{1}{\epsilon} F^{\prime}(u)\right\rangle_{2} \\
& =\left\langle\nabla u_{t}, \epsilon \nabla u\right\rangle_{2}+\left\langle u_{t}, \frac{1}{\epsilon} F^{\prime}(u)\right\rangle_{2} \\
& =\frac{d}{d t} E(u),
\end{aligned}
$$

since $u$ satisfies Neumann boundary conditions. Therefore we get

$$
\frac{d}{d t} E(u)=-\int_{\Omega}|\nabla K(u)|^{2} d x+\langle\lambda(f-u),-\epsilon \Delta u\rangle_{2}+\left\langle\lambda(f-u), \frac{1}{\epsilon} F^{\prime}(u)\right\rangle_{2} .
$$


Since $F(u)$ is bounded from below, we only have to show that $E(u)$ is uniformly bounded on $[0, T]$, and we automatically have that $|\nabla u|$ is uniformly bounded on $[0, T]$. We start with the last term, and recall the following bounds on $F^{\prime}(u)$ (cf. [67]): There exist positive constants $C_{1}, C_{2}$ such that

$$
F^{\prime}(s) s \geq C_{1} s^{2}-C_{2}, \quad \forall s \in \mathbb{R}
$$

and, for every $\delta>0$, there exists a constant $C_{3}$ such that

$$
\left|F^{\prime}(s)\right| \leq \delta C_{1} s^{2}+C_{3}(\delta), \quad \forall s \in \mathbb{R}
$$

Using the last two estimates we obtain the following:

$$
\begin{aligned}
\left\langle\lambda(f-u), \frac{1}{\epsilon} F^{\prime}(u)\right\rangle_{2}= & \frac{\lambda_{0}}{\epsilon} \int_{\Omega \backslash D} F^{\prime}(u) f d x-\frac{\lambda_{0}}{\epsilon} \int_{\Omega \backslash D} F^{\prime}(u) u d x \\
\leq & \frac{\lambda_{0}}{\epsilon} \int_{\Omega \backslash D}\left|F^{\prime}(u)\right| d x \cdot\|f\|_{L^{\infty}(\Omega)}-\frac{\lambda_{0} C_{1}}{\epsilon} \int_{\Omega \backslash D} u^{2} d x+\frac{\lambda_{0} C_{2}|\Omega \backslash D|}{\epsilon} \\
\leq & \lambda_{0} C(f, \Omega)\left(\delta \frac{C_{1}}{\epsilon} \int_{\Omega \backslash D} u^{2} d x+\frac{C_{3}(\delta)|\Omega \backslash D|}{\epsilon}\right)-\frac{\lambda_{0} C_{1}}{\epsilon} \int_{\Omega \backslash D} u^{2} d x \\
& \quad+\frac{\lambda_{0} C_{2}|\Omega \backslash D|}{\epsilon} \\
\leq & -\frac{\lambda_{0} C_{1}}{\epsilon}(1-\delta C(f, \Omega)) \int_{\Omega \backslash D} u^{2} d x+C\left(\lambda_{0}, \epsilon, \delta, \Omega, D, f\right),
\end{aligned}
$$

where we choose $\delta<1 / C(f, \Omega)$. Therefore integrating (3.14) over the time interval $[0, T]$ results in

$$
\begin{aligned}
\int_{0}^{T} \frac{d}{d t} E(u(t)) d t \leq & \int_{0}^{T}-\int_{\Omega}|\nabla K(u)|^{2} d x d t+\int_{0}^{T}\langle\lambda(f-u),-\epsilon \Delta u\rangle_{2} d t \\
& -\frac{\lambda_{0} C_{1}}{\epsilon}(1-\delta C(f, \Omega)) \int_{0}^{T} \int_{\Omega \backslash D} u^{2} d x d t+T \cdot C\left(\lambda_{0}, \epsilon, \delta, \Omega, D, f\right) .
\end{aligned}
$$

Next we consider the second term on the right side of the last inequality. From Theorem 4.1 in [13] we know that a solution $u$ of $(1.4)$ is an element in $L^{2}\left(0, T ; H^{2}(\Omega)\right)$ for all $T>0$. Hence $\Delta u \in L^{2}\left(0, T ; L^{2}(\Omega)\right)$ and the second term is bounded by a constant depending on $T$. Consequently, for each $0 \leq t \leq T$, we get

$$
\begin{aligned}
E(u(t)) \leq E(u(0))+ & C(T)+T \cdot C\left(\lambda_{0}, \epsilon, \delta, \Omega, D, f\right) \\
& -\int_{0}^{T}\left[\int_{\Omega}|\nabla K(u)|^{2} d x+\frac{\lambda_{0} C_{1}}{\epsilon}(1-\delta C(f, \Omega)) \int_{\Omega \backslash D} u^{2} d x\right] d t,
\end{aligned}
$$

and with this, for a fixed $T>0$, that $|\nabla u|$ is uniformly bounded in $[0, T]$.

3.2. Numerical results. In our computations the optimal $\Delta t$ turned out to be $\Delta t=1$ or 10 (depending also on the size of $\epsilon$ and $\lambda_{0}$ ). Numerical results of the above scheme are presented in Figures 3.1, 3.2 and 3.3. In all of the examples we follow the procedure of [13], i.e., the inpainted image is computed in a two step process. In the first step Cahn-Hilliard inpainting is solved with a rather large value of $\epsilon$, e.g., $\epsilon=0.1$, until the numerical scheme is close to steady state. In this step the level lines are continued into the missing domain. In a second step, the result of the 

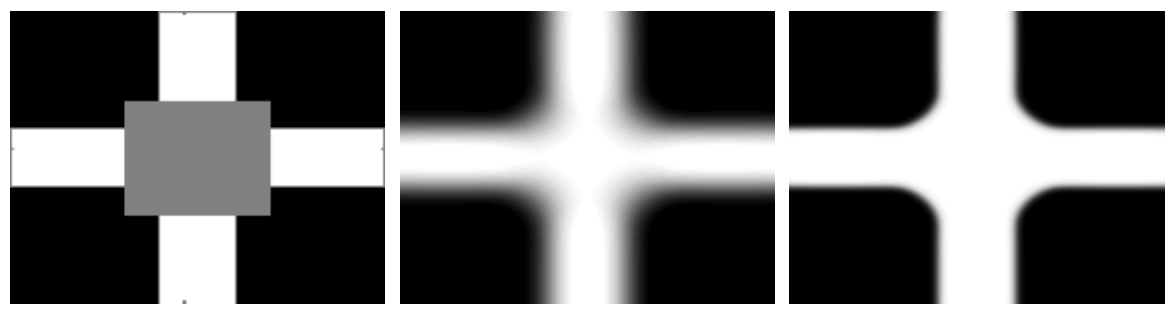

FIG. 3.1. Binary image with unknown center and the solution of Cahn-Hilliard inpainting with $\lambda_{0}=10^{5}$ and switching $\epsilon$ value: $u(600)$ with $\epsilon=0.1, u(1000)$ with $\epsilon=0.01$
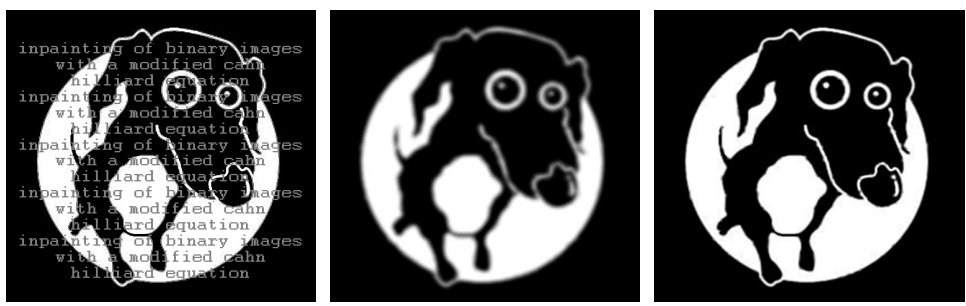

FIG. 3.2. Text removal from a binary image: the solution of Cahn-Hilliard inpainting with $\lambda_{0}=10^{9}$ and switching $\epsilon$ value: $u(200)$ with $\epsilon=0.8, u(500)$ with $\epsilon=0.01$

first step is put as an initial condition into the scheme for a small $\epsilon$, e.g., $\epsilon=0.01$, in order to sharpen the contours of the image contents. The reason for this two step procedure is twofold. First of all in [14] the authors give numerical evidence that the steady state of the modified Cahn-Hilliard Equation (1.4) is not unique, i.e., it is dependent on the initial condition inside of the inpainting domain. As a consequence, computing the inpainted image by the application of Cahn-Hilliard inpainting with a small $\epsilon$ only, might not extend the level lines into the missing domain as desired. See also [14] for a bifurcation diagram based on the numerical computations of the authors. The second reason for solving Cahn-Hilliard inpainting in two steps is that it is computationally less expensive. Solving the above time-marching scheme for, e.g., $\epsilon=0.1$ is faster than solving it for $\epsilon=0.01$. This is because of the damping introduced by $C_{1}$, i.e., $\epsilon$, into the scheme; cf. (3.2). All numerical examples presented here have been computed in orders of 10 seconds on a $1.86 \mathrm{GHz}$ processor with 1 GB RAM. For a further discussion on computational times for the convexity splitting method applied to Cahn-Hilliard inpainting we refer to [13].

One possible generalization of Cahn-Hilliard inpainting for grayscale images is to split the grayscale image bit-wise into channels

$$
u(x) \leadsto \sum_{k=1}^{K} u_{k}(x) 2^{-(k-1)},
$$

where $K>0$. The Cahn-Hilliard inpainting approach is then applied to each binary channel $u_{k}$ separately, compare Figure 3.5. At the end of the inpainting process the channels are assembled again and the result is the inpainted grayvalue image in lower grayvalue resolution, compare Figure 3.4. In Figure 3.6 the application of bitwise Cahn-Hilliard inpainting for the restoration of satellite images of roads is 

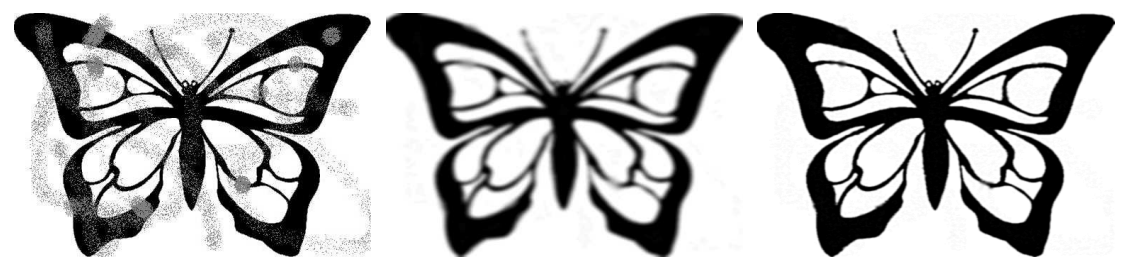

FIG. 3.3. Vandalized binary image and the solution of Cahn-Hilliard inpainting with $\lambda_{0}=10^{9}$ and switching $\epsilon$ value: $u(800)$ with $\epsilon=0.8, u(1600)$ with $\epsilon=0.01$

demonstrated. One can imagine that the black dots in the first picture represent trees that cover parts of the road. The idea of bitwise binary inpainting is proposed in [30] for the inpainting with wavelets based on the Allen-Cahn energy.
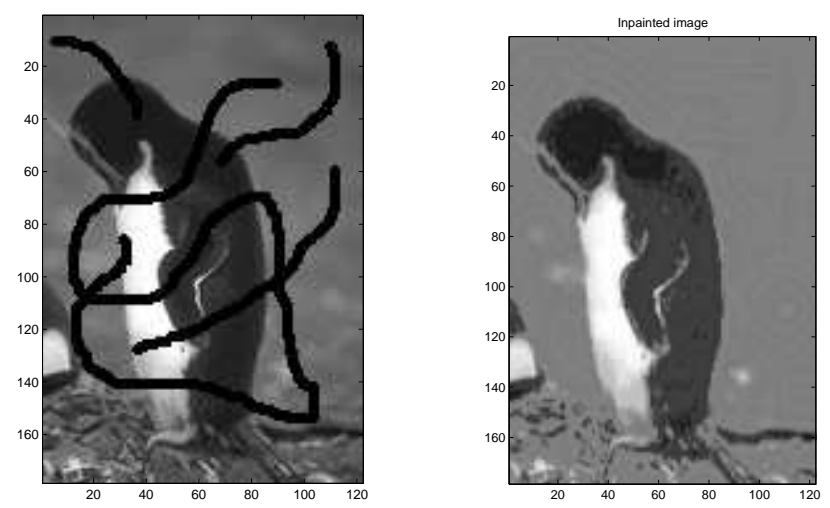

FIG. 3.4. Cahn-Hilliard bitwise inpainting with $K=8$ binary channels $\left(\lambda_{0}=10^{8}\right.$, with $\epsilon=0.1$ until $t=800$ and $\epsilon=0.01$ until $t=1200$ )

\section{4. $\mathrm{TV}-\mathrm{H}^{-1}$ inpainting}

In this section we discuss convexity splitting for $\mathrm{TV}-\mathrm{H}^{-1}$ inpainting (1.5). To avoid numerical and theoretical difficulties we approximate an element $p$ in the subdifferential of the total variation functional $T V(u)$ by a smoothed version of $\nabla \cdot(\nabla u /|\nabla u|)$, the square root regularization for instance. With the latter regularization the smoothed version of (1.5) reads as

$$
u_{t}=-\Delta \nabla \cdot\left(\frac{\nabla u}{\sqrt{|\nabla u|^{2}+\delta^{2}}}\right)+\lambda(f-u),
$$

with $0<\delta \ll 1$. In contrast to its second-order analogue, the well-posedness of (1.5) strongly depends on the smoothing used for $\nabla \cdot(\nabla u /|\nabla u|)$. In fact there are smoothing functions for which (1.5) produces singularities in finite time. This is caused by the lack of maximum principles which in the second-order case guarantee the wellposedness for all smooth monotone regularizations. In [16] the authors consider (1.5) with $\lambda=\lambda_{0}$ in all of $\Omega$, i.e., the fourth-order analogue to TV-L ${ }^{2}$ denoising, which was originally introduced in [58]. They prove global well-posedness, in one space 

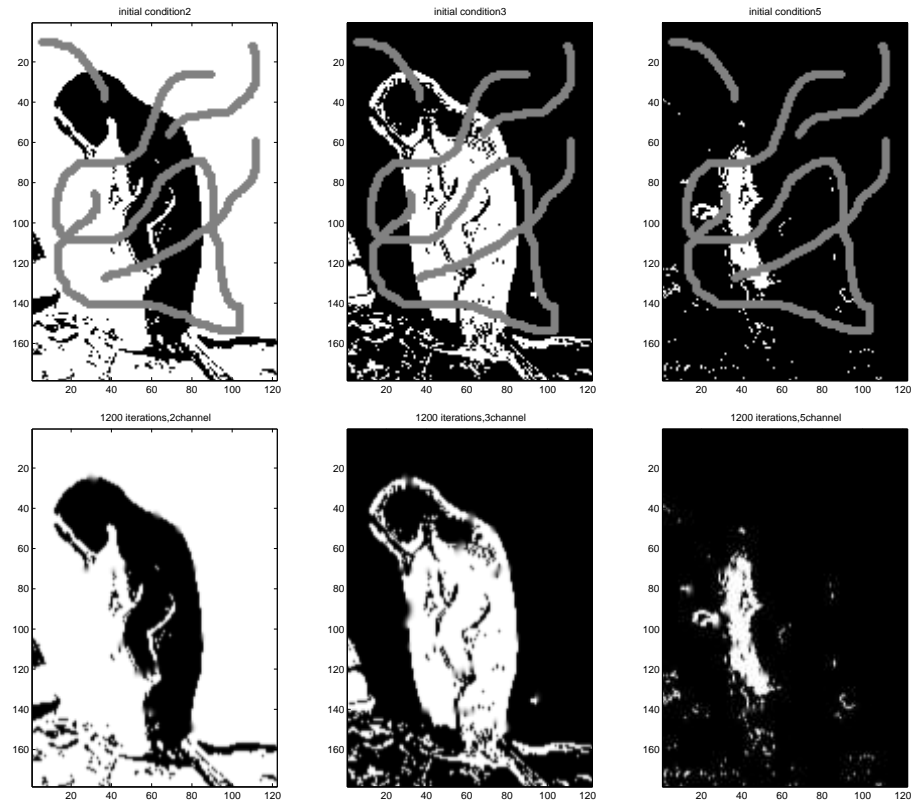

FIG. 3.5. The given image (first row) and the Cahn-Hilliard inpainting result (second row) for the channels 2,3 and 5 .
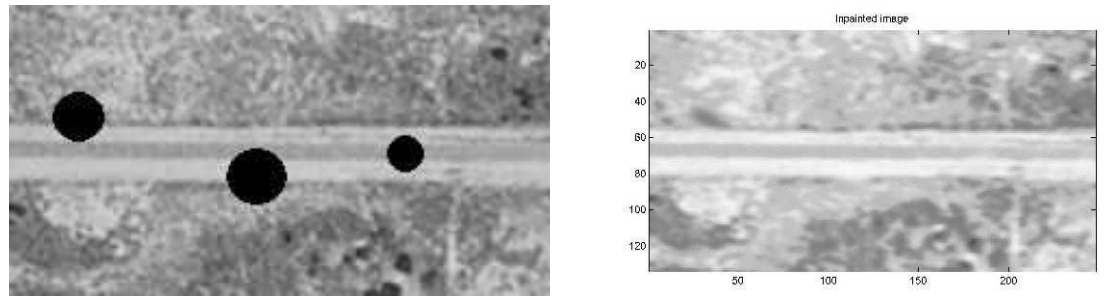

FIG. 3.6. Bitwise Cahn-Hilliard inpainting with $K=8$ binary channels applied to road restoration

dimension and for smooth initial data, for the arctan regularization

$$
\left(\frac{2}{\pi} \arctan \left(u_{x} / \delta\right)\right)_{x}
$$

where $0<\delta \ll 1$. For the square root smoothing

$$
\left(\frac{u_{x}}{\sqrt{\left|u_{x}\right|^{2}+\delta^{2}}}\right)_{x}
$$

they conjecture, supported by empirical evidence, that singularities occur in infinite time, not finite time. The behavior of the fourth-order PDE in one dimension is also relevant for two-dimensional images since a lot of structure involves edges which are one-dimensional objects. In two dimensions similar results are much more difficult to obtain, since energy estimates and the Sobolev lemma involved in its proof might not 
hold in higher dimensions anymore. We also note that in [19] the authors prove the existence of a weak stationary solution for (1.5) in two space dimensions.

In the following we present the convexity splitting method applied to (1.5) for both the square root and the arctan regularization. Similarly to the convexity splitting for Cahn-Hilliard inpainting, we propose the following splitting for the TV- $\mathrm{H}^{-1}$ inpainting equation. The regularizing term in (1.5) can be modeled by a gradient flow in $H^{-1}$ of the energy

$$
E_{1}(u)=\int_{\Omega}|\nabla u| d x
$$

where $|\nabla u|$ is replaced by its regularized version, e.g., $\sqrt{|\nabla u|^{2}+\delta^{2}}, \delta>0$. We split $E_{1}$ as $E_{1 c}-E_{1 e}$, with

$$
E_{1 c}(u)=\int_{\Omega} \frac{C_{1}}{2}|\nabla u|^{2} d x, \text { and } E_{1 e}(u)=\int_{\Omega}-|\nabla u|+\frac{C_{1}}{2}|\nabla u|^{2} d x .
$$

The fitting term is split into $E_{2}=E_{2 c}-E_{2 e}$, analogous to Cahn-Hilliard inpainting. The resulting time-stepping scheme is given by

$$
\begin{gathered}
\frac{U_{k+1}-U_{k}}{\Delta t}+C_{1} \Delta \Delta U_{k+1}+C_{2} U_{k+1}=C_{1} \Delta \Delta U_{k}-\Delta\left(\nabla \cdot\left(\frac{\nabla U_{k}}{\left|\nabla U_{k}\right|}\right)\right) \\
+C_{2} U_{k}+\lambda\left(f-U_{k}\right) .
\end{gathered}
$$

We assume that $U_{k+1}$ satisfies zero Neumann boundary conditions and use the DCT to solve (4.4).

The constants $C_{1}$ and $C_{2}$ have to be chosen such that $E_{1 c}, E_{1 e}, E_{2 c}, E_{2 e}$ are all strictly convex. In the following we demonstrate how to compute the appropriate constants. Let us consider $C_{1}$ first. The functional $E_{1 c}$ is strictly convex for all $C_{1}>0$. The choice of $C_{1}$ for the convexity of $E_{1 e}$ depends on the regularization of the total variation we are using. We use the square regularization (4.3), i.e., instead of $|\nabla u|$ we have

$$
\int G(|\nabla u|) d x, \text { with } G(s)=\sqrt{s^{2}+\delta^{2}} .
$$

Setting $y=|\nabla u|$ we have to choose $C_{1}$ such that $\frac{C_{1}}{2} y^{2}-G(y)$ is convex. The convexity condition for the second derivative gives us that

$$
C_{1}>G^{\prime \prime}(y) \Longleftrightarrow C_{1}>\frac{\delta^{2}}{\left(\delta^{2}+y^{2}\right)^{3 / 2}} \Longleftrightarrow C_{1}>\frac{1}{\delta}
$$

is sufficient as $\frac{\delta^{2}}{\left(\delta^{2}+y^{2}\right)^{3 / 2}}$ has its maximum value at $y=0$. In the one dimensional case, we would like to compare this with the arctan regularization (4.2), i.e., replacing $\frac{u_{x}}{\left|u_{x}\right|}$ by $\frac{2}{\pi} \arctan \left(\frac{u_{x}}{\delta}\right)$ as proposed in [16]. Here the convexity condition for the second derivative reads

$$
C_{1} \pm \frac{d}{d s}\left(\frac{2}{\pi} \arctan \left(\frac{s}{\delta}\right)\right)>0
$$

The \pm sign results from the absent absolute value in the regularization definition. We obtain

$$
C_{1} \pm \frac{2}{\pi} \frac{1}{\delta\left(1+s^{2} / \delta^{2}\right)}>0
$$


The inequality with a plus sign instead of \pm is true for all constants $C_{1}>0$. In the other case we obtain

$$
C_{1}>\frac{2}{\pi} \frac{\delta}{\delta^{2}+s^{2}}
$$

which is fulfilled for all $s \in \mathbb{R}$ if $C_{1}>\frac{2}{\delta \pi}$. Note that this condition is almost the same as in the case of the square regularization.

Now we consider $E_{2}=E_{2 c}-E_{2 e}$. The functional $E_{2 c}$ is strictly convex if $C_{2}>0$. For the convexity of $E_{2 e}$ we rewrite

$$
\begin{aligned}
E_{2 e}(u) & =\frac{1}{2} \int_{\Omega}-\lambda(f-u)^{2}+C_{2}|u|^{2} d x \\
& =\int_{D} \frac{C_{2}}{2}|u|^{2} d x+\int_{\Omega \backslash D}-\frac{\lambda_{0}}{2}(f-u)^{2}+\frac{C_{2}}{2}|u|^{2} d x \\
& =\int_{D} \frac{C_{2}}{2}|u|^{2} d x+\int_{\Omega \backslash D}\left(\frac{C_{2}}{2}-\frac{\lambda_{0}}{2}\right)|u|^{2}+\lambda_{0} f u-\frac{\lambda_{0}}{2}|f|^{2} .
\end{aligned}
$$

This is convex for $C_{2}>\lambda_{0}$, e.g., with $C_{2}=\lambda_{0}+1$ we can write

$$
E_{2 e}(u)=\int_{D} \frac{C_{2}}{2}|u|^{2} d x+\int_{\Omega \backslash D}\left(\frac{1}{2} u+\lambda_{0} f\right)^{2}-\left(\lambda_{0}^{2}+\frac{\lambda_{0}}{2}\right)|f|^{2} d x .
$$

4.1. Rigorous estimates for the scheme. As in Section 3.1 for CahnHilliard inpainting, we proceed with a more detailed analysis of (4.4). Throughout this section we consider the square-root regularization of the total variation both in our numerical scheme and in the continuous evolution Equation (1.5). Note that similar results are true for other monotone regularizers such as the arctan smoothing. Our results are summarized in the following theorem.

THEOREM 4.1. Let $u$ be the exact solution of (4.1) and $u_{k}=u(k \Delta t)$ be the exact solution at time $k \Delta t$ for a time step $\Delta t>0$ and $k \in \mathbb{N}$. Let $U_{k}$ be the kth iterate of (4.4) with constants $C_{1}>1 / \delta, C_{2}>\lambda_{0}$. Then the following statements are true:

(i) Under the assumption that $\left\|u_{t t}\right\|_{-1}$ and $\left\|\nabla \Delta u_{t}\right\|_{2}$ are bounded, the numerical scheme (4.4) is consistent with the continuous Equation (1.5) and of order one in time.

(ii) The solution sequence $U_{k}$ is bounded on a finite time interval $[0, T]$, for all $\Delta t>0$. In particular, for $k \Delta t \leq T, T>0$ fixed, we have for every $\Delta t>0$,

$$
\left\|\nabla U_{k}\right\|_{2}^{2}+\Delta t K_{1}\left\|\nabla \Delta U_{k}\right\|_{2}^{2} \leq e^{K_{2} T}\left(\left\|\nabla U_{0}\right\|_{2}^{2}+\Delta t K_{1}\left\|\nabla \Delta U_{0}\right\|_{2}^{2}+\Delta t T C\left(\Omega, D, \lambda_{0}, f\right)\right)
$$

for suitable constants $K_{1}, K_{2}$, and a constant $C$, which depends on $\Omega, D, \lambda_{0}, f$ only.

(iii) Let $e_{k}=u_{k}-U_{k}$. For smooth solutions $u_{k}$ and $U_{k}$, the error $e_{k}$ converges to zero as $\Delta t \rightarrow 0$. In particular, for $k \Delta t \leq T, T>0$ fixed, we have

$$
\left\|\nabla e_{k}\right\|_{2}^{2}+\Delta t M_{1}\left\|\nabla \Delta e_{k}\right\|_{2}^{2} \leq \frac{T}{M_{2}} e^{M_{3} T}(\Delta t)^{2}
$$

for suitable positive constants $M_{1}, M_{2}$ and $M_{3}$. 
REMARK 4.1. For the convergence result in Theorem 4.1 (iii) we assume that smooth solutions to both the continuous in time problem and the discrete in time approximation exist. The validity of this assumption is not known in general. Note however that the global regularity results are known in 1D for the arctan smoothing [16]. Moreover, our numerical results show no indication of singularities in 2D. Therefore, it is not unreasonable to analyze the convergence under these assumptions.

The proof of Theorem 4.1 is split into the three separate Propositions 4.2-4.4.

Proposition 4.2 (Consistency (i)). Under the same assumptions as in Theorem 4.1 and in particular under the assumption that $\left\|u_{t t}\right\|_{-1}$ and $\left\|\nabla \Delta u_{t}\right\|_{2}$ are bounded, the numerical scheme (4.4) is consistent with the continuous Equation (4.1) with $\left\|\tau_{k}\right\|_{-1}=O(\Delta t)$ as $\Delta t \rightarrow 0$, where $\tau_{k}$ is the local truncation error.

Proof. The local truncation error is defined over a time step as satisfying

$$
\tau_{k}=\tau_{k}^{1}+\tau_{k}^{2},
$$

where

$$
\tau_{k}^{1}=\frac{u_{k+1}-u_{k}}{\Delta t}-u_{t}(k \Delta t), \quad \tau_{k}^{2}=C_{1} \Delta^{2}\left(u_{k+1}-u_{k}\right)+C_{2}\left(u_{k+1}-u_{k}\right),
$$

i.e.,

$$
\begin{aligned}
\tau_{k}=\frac{u_{k+1}-u_{k}}{\Delta t}+\Delta\left(\nabla \cdot\left(\frac{\nabla u_{k}}{\sqrt{\left|\nabla u_{k}\right|^{2}+\delta^{2}}}\right)\right)-\lambda\left(f-u_{k}\right) \\
+C_{1} \Delta^{2}\left(u_{k+1}-u_{k}\right)+C_{2}\left(u_{k+1}-u_{k}\right) .
\end{aligned}
$$

Using standard Taylor series arguments and assuming that $\left\|u_{t t}\right\|_{-1},\left\|\nabla \Delta u_{t}\right\|_{2}$ and $\left\|u_{t}\right\|_{2}$ are bounded we deduce that

$$
\left\|\tau_{k}\right\|_{-1}=O(\Delta t) \quad \text { for } \Delta t \rightarrow 0
$$

Proposition 4.3 (Unconditional stability (ii)). Under the same assumptions as in Theorem 4.1 the solution sequence $U_{k}$ fulfills (4.5). This gives boundedness of the solution sequence on $[0, T]$.

Proof. If we multiply (4.4) with $-\Delta U_{k+1}$ and integrate over $\Omega$ we obtain

$$
\begin{aligned}
& \frac{1}{\Delta t}\left(\left\|\nabla U_{k+1}\right\|_{2}^{2}-\left\langle\nabla U_{k}, \nabla U_{k+1}\right\rangle_{2}\right)+C_{2}\left\|\nabla U_{k+1}\right\|_{2}^{2}+C_{1}\left\|\nabla \Delta U_{k+1}\right\|_{2}^{2} \\
= & \left\langle\Delta \nabla \cdot\left(\frac{\nabla U_{k}}{\sqrt{\left|\nabla U_{k}\right|^{2}+\delta^{2}}}\right), \Delta U_{k+1}\right\rangle_{2}+C_{1}\left\langle\nabla \Delta U_{k}, \nabla \Delta U_{k+1}\right\rangle_{2} \\
+ & \left\langle\nabla\left(\lambda\left(f-U_{k}\right)\right), \nabla U_{k+1}\right\rangle_{2}+C_{2}\left\langle\nabla U_{k}, \nabla U_{k+1}\right\rangle_{2} .
\end{aligned}
$$

Applying Young's inequality to the inner products on the right and estimating

$$
\left\|\nabla \lambda\left(f-U_{k}\right)\right\|_{2}^{2} \leq 2 \lambda_{0}^{2}\left\|\nabla U_{k}\right\|_{2}^{2}+C\left(\Omega, D, \lambda_{0}, f\right)
$$


results in

$$
\begin{aligned}
& \frac{1}{2 \Delta t}\left(\left\|\nabla U_{k+1}\right\|_{2}^{2}-\left\|\nabla U_{k}\right\|_{2}^{2}\right)+C_{2}\left\|\nabla U_{k+1}\right\|_{2}^{2}+C_{1}\left\|\nabla \Delta U_{k+1}\right\|_{2}^{2} \\
\leq & \left\langle\Delta \nabla \cdot\left(\frac{\nabla U_{k}}{\sqrt{\left|\nabla U_{k}\right|^{2}+\delta^{2}}}\right), \Delta U_{k+1}\right\rangle_{2}+\frac{C_{1}}{\delta_{1}}\left\|\nabla \Delta U_{k}\right\|_{2}^{2}+C_{1} \delta_{1}\left\|\nabla \Delta U_{k+1}\right\|_{2}^{2} \\
& +\frac{2 \lambda_{0}^{2}}{\delta_{2}}\left\|\nabla U_{k}\right\|_{2}^{2}+\delta_{2}\left\|\nabla U_{k+1}\right\|_{2}^{2}+\frac{C_{2}}{\delta_{3}}\left\|\nabla U_{k}\right\|_{2}^{2}+C_{2} \delta_{3}\left\|\nabla U_{k+1}\right\|_{2}^{2}+C\left(\Omega, D, \lambda_{0}, f\right) .
\end{aligned}
$$

Now, the first term on the right side of the inequality can be estimated as follows

$$
\begin{aligned}
& \left\langle\Delta \nabla \cdot\left(\frac{\nabla U_{k}}{\sqrt{\left|\nabla U_{k}\right|^{2}+\delta^{2}}}\right), \Delta U_{k+1}\right\rangle_{2}=-\left\langle\nabla \nabla \cdot\left(\frac{\nabla U_{k}}{\sqrt{\left|\nabla U_{k}\right|^{2}+\delta^{2}}}\right), \nabla \Delta U_{k+1}\right\rangle_{2} \\
& \leq \frac{1}{\delta_{4}}\left\|\nabla \nabla \cdot\left(\frac{\nabla U_{k}}{\sqrt{\left|\nabla U_{k}\right|^{2}+\delta^{2}}}\right)\right\|_{2}^{2}+\delta_{4}\left\|\nabla \Delta U_{k+1}\right\|_{2}^{2} .
\end{aligned}
$$

Applying Poincaré's and Cauchy's inequality to the first term leads to

$$
\left\|\nabla \nabla \cdot\left(\frac{\nabla U_{k}}{\sqrt{\left|\nabla U_{k}\right|^{2}+\delta^{2}}}\right)\right\|_{2}^{2} \leq \frac{C}{\delta}\left(\left\|\nabla U_{k}\right\|_{2}^{2}+\left\|\Delta U_{k}\right\|_{2}^{2}+\left\|\nabla \Delta U_{k}\right\|_{2}^{2}\right) .
$$

Interpolating the $L^{2}$ norm of $\Delta u$ by the $L^{2}$ norms of $\nabla u$ and $\nabla \Delta u$, we obtain

$$
\begin{aligned}
&\left(\frac{1}{2 \Delta t}+C_{2}\left(1-\delta_{3}\right)-\delta_{2}\right)\left\|\nabla U_{k+1}\right\|_{2}^{2}+\left(C_{1}\left(1-\delta_{1}\right)-\delta_{4}\right)\left\|\nabla \Delta U_{k+1}\right\|_{2}^{2} \\
& \leq\left(\frac{1}{2 \Delta t}+\frac{2 \lambda_{0}^{2}}{\delta_{2}}+\frac{C_{2}}{\delta_{3}}+\frac{C(1 / \delta, \Omega)}{\delta_{4}}\right)\left\|\nabla U_{k}\right\|_{2}^{2}+\left(\frac{C_{1}}{\delta_{1}}+\frac{C(1 / \delta, \Omega)}{\delta_{4}}\right)\left\|\nabla \Delta U_{k}\right\|_{2}^{2} \\
&+C\left(\Omega, D, \lambda_{0}, f\right) .
\end{aligned}
$$

For $\delta_{i}=1 / 2, i=1, \ldots, 4$ we obtain

$$
\begin{aligned}
& \left(\frac{1}{2 \Delta t}+\frac{C_{2}-1}{2}\right)\left\|\nabla U_{k+1}\right\|_{2}^{2}+\frac{C_{1}-1}{2}\left\|\nabla \Delta U_{k+1}\right\|_{2}^{2} \\
\leq & \left(\frac{1}{2 \Delta t}+4 \lambda_{0}^{2}+2\left(C_{2}+C\right)\right)\left\|\nabla U_{k}\right\|_{2}^{2}+2\left(C_{1}+C\right)\left\|\nabla \Delta U_{k}\right\|_{2}^{2}+C\left(\Omega, D, \lambda_{0}, f\right) .
\end{aligned}
$$

Since $C_{1}$ and $C_{2}$ are chosen such that $C_{1}>1 / \delta>1$ and $C_{2}>\lambda_{0}>1$, the coefficients in the inequality above are positive. The rest of the proof is similar to the proof of Proposition 3.3. We multiply the inequality by $2 \Delta t$ and set

$$
C_{a}=1+\Delta t\left(C_{2}-1\right), \quad C_{b}=C_{1}-1, \quad C_{c}=1+2 \Delta t\left(4 \lambda_{0}^{2}+2\left(C_{2}+C\right)\right), \quad C_{d}=4\left(C_{1}+C\right) .
$$

We obtain

$$
C_{a}\left\|\nabla U_{k+1}\right\|_{2}^{2}+\Delta t C_{b}\left\|\nabla \Delta U_{k+1}\right\|_{2}^{2} \leq C_{c}\left\|\nabla U_{k}\right\|_{2}^{2}+\Delta t C_{d}\left\|\nabla \Delta U_{k}\right\|_{2}^{2}+2 \Delta t C\left(\Omega, D, \lambda_{0}, f\right) .
$$


Dividing by $C_{a}$ (which is $>0$ ) we have

$$
\left\|\nabla U_{k+1}\right\|_{2}^{2}+\Delta t \frac{C_{b}}{C_{a}}\left\|\nabla \Delta U_{k+1}\right\|_{2}^{2} \leq \frac{C_{c}}{C_{a}}\left\|\nabla U_{k}\right\|_{2}^{2}+\Delta t \frac{C_{d}}{C_{a}}\left\|\nabla \Delta U_{k}\right\|_{2}^{2}+\frac{2}{C_{a}} \Delta t C\left(\Omega, D, \lambda_{0}, f\right) .
$$

We rewrite the right hand side of the inequality such that

$$
\begin{aligned}
& \left\|\nabla U_{k+1}\right\|_{2}^{2}+\Delta t \frac{C_{b}}{C_{a}}\left\|\nabla \Delta U_{k+1}\right\|_{2}^{2} \\
\leq & \frac{C_{c} C_{d}}{C_{a}}\left(\frac{1}{C_{d}}\left\|\nabla U_{k}\right\|_{2}^{2}+\Delta t \frac{1}{C_{c}}\left\|\nabla \Delta U_{k}\right\|_{2}^{2}\right)+\frac{2}{C_{a}} \Delta t C\left(\Omega, D, \lambda_{0}, f\right) .
\end{aligned}
$$

Since $C_{d}>1$ we can multiply the first term within the brackets on the right hand side of the inequality with $C_{d}$ and will only get something which is larger or equal. For the same reason we can multiply the second term within the brackets with

$$
1<\frac{C_{c} C_{b}}{C_{a}}=\frac{\left(1+2 \Delta t\left(4 \lambda_{0}^{2}+2\left(C_{2}+C\right)\right)\right)\left(C_{1}-1\right)}{1+\Delta t\left(C_{2}-1\right)}
$$

and get

$$
\begin{aligned}
& \left\|\nabla U_{k+1}\right\|_{2}^{2}+\Delta t \frac{C_{b}}{C_{a}}\left\|\nabla \Delta U_{k+1}\right\|_{2}^{2} \\
\leq & \frac{C_{c} C_{d}}{C_{a}}\left(\left\|\nabla U_{k}\right\|_{2}^{2}+\Delta t \frac{C_{b}}{C_{a}}\left\|\nabla \Delta U_{k}\right\|_{2}^{2}\right)+\frac{2}{C_{a}} \Delta t C\left(\Omega, D, \lambda_{0}, f\right) .
\end{aligned}
$$

By induction it follows that

$$
\begin{aligned}
\left\|\nabla U_{k+1}\right\|_{2}^{2}+\Delta t \frac{C_{b}}{C_{a}}\left\|\nabla \Delta U_{k+1}\right\|_{2}^{2} \leq\left(\frac{C_{c} C_{d}}{C_{a}}\right)^{k} & \left(\left\|\nabla U_{0}\right\|_{2}^{2}+\Delta t \frac{C_{b}}{C_{a}}\left\|\nabla \Delta U_{0}\right\|_{2}^{2}\right) \\
& +\Delta t \sum_{i=0}^{k-1}\left(\frac{C_{c} C_{d}}{C_{a}}\right)^{i} \frac{2}{C_{a}} C\left(\Omega, D, \lambda_{0}, f\right) .
\end{aligned}
$$

Therefore we obtain for $k \Delta t \leq T$

$$
\left\|\nabla U_{k}\right\|_{2}^{2}+\Delta t \frac{C_{b}}{C_{a}}\left\|\nabla \Delta U_{k}\right\|_{2}^{2} \leq e^{K T}\left(\left\|\nabla U_{0}\right\|_{2}^{2}+\Delta t \frac{C_{b}}{C_{a}}\left\|\nabla \Delta U_{0}\right\|_{2}^{2}+\Delta t T \frac{2}{C_{a}} C\left(\Omega, D, \lambda_{0}, f\right)\right) .
$$

Finally we show that the discrete solution converges to the continuous one as $\Delta t$ tends to zero.

Proposition 4.4 (Convergence (iii)). Under the same assumptions as in Theorem 4.1 (iii) the error $e_{k}$ fulfills (4.6).

Proof. By our discrete Approximation (4.4) and the consistency computation 
(4.7), we have for $e_{k}=u_{k}-U_{k}$

$$
\begin{aligned}
& \frac{e_{k+1}-e_{k}}{\Delta t}+C_{1} \Delta^{2} e_{k+1}+C_{2} e_{k+1} \\
= & \frac{1}{\Delta t}\left(u_{k+1}-u_{k}\right)-\frac{1}{\Delta t}\left(U_{k+1}-U_{k}\right)+C_{1} \Delta^{2} u_{k+1}-C_{1} \Delta^{2} U_{k+1}+C_{2} u_{k+1}-C_{2} U_{k+1} \\
= & -\left(C_{1} \Delta^{2} U_{k}-\Delta\left(\nabla \cdot\left(\frac{\nabla U_{k}}{\sqrt{\left|\nabla U_{k}\right|^{2}+\delta^{2}}}\right)\right)+\lambda\left(f-U_{k}\right)+C_{2} U_{k}\right) \\
& -\left(\Delta\left(\nabla \cdot\left(\frac{\nabla u_{k}}{\sqrt{\left|\nabla u_{k}\right|^{2}+\delta^{2}}}\right)\right)-\lambda\left(f-u_{k}\right)-C_{1} \Delta^{2} u_{k}-C_{2} u_{k}\right)+\tau_{k} \\
= & -\left[-\Delta\left(\nabla \cdot\left(\frac{\nabla U_{k}}{\sqrt{\left|\nabla U_{k}\right|^{2}+\delta^{2}}}\right)-\nabla \cdot\left(\frac{\nabla u_{k}}{\sqrt{\left|\nabla u_{k}\right|^{2}+\delta^{2}}}\right)\right)\right. \\
& \left.+C_{1} \Delta^{2}\left(U_{k}-u_{k}\right)+C_{2}\left(U_{k}-u_{k}\right)-\lambda\left(U_{k}-u_{k}\right)\right]+\tau_{k} .
\end{aligned}
$$

Taking the inner product with $-\Delta e_{k+1}$, we have

$$
\begin{aligned}
& \frac{1}{\Delta t}\left\langle\nabla\left(e_{k+1}-e_{k}\right), \nabla e_{k+1}\right\rangle_{2}+C_{1}\left\|\nabla \Delta e_{k+1}\right\|_{2}^{2}+C_{2}\left\|\nabla e_{k+1}\right\|_{2}^{2} \\
&=\left\langle-\Delta\left(\nabla \cdot\left(\frac{\nabla U_{k}}{\sqrt{\left|\nabla U_{k}\right|^{2}+\delta^{2}}}\right)-\nabla \cdot\left(\frac{\nabla u_{k}}{\sqrt{\left|\nabla u_{k}\right|^{2}+\delta^{2}}}\right)\right), \Delta e_{k+1}\right\rangle_{2} \\
&+C_{1}\left\langle\Delta^{2}\left(U_{k}-u_{k}\right), \Delta e_{k+1}\right\rangle_{2}+\left\langle\nabla \lambda\left(U_{k}-u_{k}\right), \nabla e_{k+1}\right\rangle_{2} \\
& \quad-C_{2}\left\langle\nabla\left(U_{k}-u_{k}\right), \nabla e_{k+1}\right\rangle_{2}-\left\langle\nabla \Delta^{-1} \tau_{k}, \nabla \Delta e_{k+1}\right\rangle_{2} .
\end{aligned}
$$

Using the same arguments as in the proof of Proposition 3.4 we obtain

$$
\begin{aligned}
& \frac{1}{2 \Delta t}\left(\left\|\nabla e_{k+1}\right\|_{2}^{2}-\left\|\nabla e_{k}\right\|_{2}^{2}\right)+C_{1}\left\|\nabla \Delta e_{k+1}\right\|_{2}^{2}+C_{2}\left\|\nabla e_{k+1}\right\|_{2}^{2} \\
& \leq\left\langle-\Delta\left(\nabla \cdot\left(\frac{\nabla U_{k}}{\sqrt{\left|\nabla U_{k}\right|^{2}+\delta^{2}}}\right)-\nabla \cdot\left(\frac{\nabla u_{k}}{\sqrt{\left|\nabla u_{k}\right|^{2}+\delta^{2}}}\right)\right), \Delta e_{k+1}\right\rangle_{2} \\
&+ \frac{C_{1}}{\delta_{1}}\left\|\nabla \Delta e_{k}\right\|_{2}^{2}+C_{1} \delta_{1}\left\|\nabla \Delta e_{k+1}\right\|_{2}^{2}+\frac{\lambda_{0}^{2}}{\delta_{3}}\left\|\nabla e_{k}\right\|_{2}^{2}+\delta_{3}\left\|\nabla e_{k+1}\right\|_{2}^{2} \\
&+\frac{C_{2}}{\delta_{2}}\left\|\nabla e_{k}\right\|_{2}^{2}+C_{2} \delta_{2}\left\|\nabla e_{k+1}\right\|_{2}^{2}+\frac{1}{\delta_{4}}\left\|\tau_{k}\right\|_{-1}^{2}+\delta_{4}\left\|\nabla \Delta e_{k+1}\right\|_{2}^{2} .
\end{aligned}
$$

We consider the first term on the right side of the above inequality in detail,

$$
\begin{aligned}
& \left\langle-\Delta\left(\nabla \cdot\left(\frac{\nabla U_{k}}{\sqrt{\left|\nabla U_{k}\right|^{2}+\delta^{2}}}\right)-\nabla \cdot\left(\frac{\nabla u_{k}}{\sqrt{\left|\nabla u_{k}\right|^{2}+\delta^{2}}}\right)\right), \Delta e_{k+1}\right\rangle_{2} \\
= & \left\langle\nabla\left(\nabla \cdot\left(\frac{\nabla U_{k}}{\sqrt{\left|\nabla U_{k}\right|^{2}+\delta^{2}}}\right)-\nabla \cdot\left(\frac{\nabla u_{k}}{\sqrt{\left|\nabla u_{k}\right|^{2}+\delta^{2}}}\right)\right), \nabla \Delta e_{k+1}\right\rangle_{2} .
\end{aligned}
$$


We get

$$
\nabla \cdot\left(\frac{\nabla u}{\sqrt{|\nabla u|^{2}+\delta^{2}}}\right)=\frac{\Delta u}{\sqrt{|\nabla u|^{2}+\delta^{2}}}-\frac{u_{x}^{2} u_{x x}+2 u_{x} u_{y} u_{x y}+u_{y}^{2} u_{y y}}{\left(|\nabla u|^{2}+\delta^{2}\right)^{3 / 2}}
$$

Next, we apply the gradient to this expression and obtain

$$
\begin{aligned}
& \nabla\left(\nabla \cdot\left(\frac{\nabla u}{\sqrt{|\nabla u|^{2}+\delta^{2}}}\right)\right) \\
&=\frac{\nabla \Delta u}{\sqrt{|\nabla u|^{2}+\delta^{2}}}-\frac{\Delta u}{2\left(|\nabla u|^{2}+\delta^{2}\right)^{3 / 2}} \cdot \nabla\left(|\nabla u|^{2}\right)-\frac{\nabla\left(u_{x}^{2} u_{x x}+2 u_{x} u_{y} u_{x y}+u_{y}^{2} u_{y y}\right)}{\left(|\nabla u|^{2}+\delta^{2}\right)^{3 / 2}} \\
&+\frac{3\left(u_{x}^{2} u_{x x}+2 u_{x} u_{y} u_{x y}+u_{y}^{2} u_{y y}\right)}{2\left(|\nabla u|^{2}+\delta^{2}\right)^{5 / 2}} \cdot \nabla\left(|\nabla u|^{2}\right),
\end{aligned}
$$

where

$$
\nabla\left(|\nabla u|^{2}\right)=\left(\begin{array}{c}
2 \nabla u \cdot\left(\begin{array}{l}
u_{x x} \\
u_{y x}
\end{array}\right) \\
2 \nabla u \cdot\left(\begin{array}{l}
u_{x y} \\
u_{y y}
\end{array}\right)
\end{array}\right)=2\left(\begin{array}{l}
u_{x} u_{x x}+u_{y} u_{y x} \\
u_{x} u_{x y}+u_{y} u_{y y}
\end{array}\right)
$$

and

$$
\begin{array}{r}
\nabla\left(u_{x}^{2} u_{x x}+2 u_{x} u_{y} u_{x y}+u_{y}^{2} u_{y y}\right)=2\left(u_{x} u_{x x}+u_{y} u_{x y}\right) \nabla u_{x}+2\left(u_{x} u_{x y}+u_{y} u_{y y}\right) \nabla u_{y} \\
+u_{x}^{2} \nabla u_{x x}+2 u_{x} u_{y} \nabla u_{x y}+u_{y}^{2} \nabla u_{y y} .
\end{array}
$$

Reordering the involved terms we have

$$
\begin{aligned}
& \nabla\left(\nabla \cdot\left(\frac{\nabla u}{\sqrt{|\nabla u|^{2}+\delta^{2}}}\right)\right) \\
= & H_{1}(\nabla u) \cdot \nabla \Delta u+H_{2}\left(u_{x}, u_{y}, u_{x x}, u_{x y}, u_{y y}\right) \cdot \nabla u_{x}+H_{3}\left(u_{x}, u_{y}, u_{x x}, u_{x y}, u_{y y}\right) \cdot \nabla u_{y} \\
& +H_{4}\left(u_{x}, u_{y}\right) \cdot \nabla u_{x x}+H_{5}\left(u_{x}, u_{y}\right) \cdot \nabla u_{x y}+H_{6}\left(u_{x}, u_{y}\right) \cdot \nabla u_{y y},
\end{aligned}
$$


where

$$
\begin{aligned}
H_{1}(\nabla u) & =\frac{1}{\sqrt{|\nabla u|^{2}+\delta^{2}}}, \\
H_{2}\left(u_{x}, u_{y}, u_{x x}, u_{x y}, u_{y y}\right) & =-\left(\frac{\Delta u u_{x}+2\left(u_{x} u_{x x}+u_{y} u_{x y}\right)}{\left(|\nabla u|^{2}+\delta^{2}\right)^{3 / 2}}\right. \\
& \left.-\frac{3\left(u_{x}^{2} u_{x x}+2 u_{x} u_{y} u_{x y}+u_{y}^{2} u_{y y}\right) u_{x}}{\left(|\nabla u|^{2}+\delta^{2}\right)^{5 / 2}}\right), \\
H_{3}\left(u_{x}, u_{y}, u_{x x}, u_{x y}, u_{y y}\right) & =-\left(\frac{\Delta u u_{y}+2\left(u_{x} u_{x y}+u_{y} u_{y y}\right)}{\left(|\nabla u|^{2}+\delta^{2}\right)^{3 / 2}}\right. \\
H_{4}\left(u_{x}, u_{y}\right) & \left.=-\frac{3\left(u_{x}^{2} u_{x x}+2 u_{x} u_{y} u_{x y}+u_{y}^{2} u_{y y}\right) u_{y}}{\left(|\nabla u|^{2}+\delta^{2}\right)^{5 / 2}}\right), \\
H_{5}\left(u_{x}, u_{y}\right) & =-\frac{u_{x}^{2}}{\left(|\nabla u|^{2}+\delta^{2}\right)^{3 / 2}}, \\
H_{6}\left(u_{x}, u_{y}\right) & =-\frac{\left.u_{y}^{2} \delta^{2}\right)^{3 / 2}}{\left(|\nabla u|^{2}+\delta^{2}\right)^{3 / 2}} .
\end{aligned}
$$

Now we are going to insert this into (4.10). For ease of notation we suppress the time index $k$ for now, i.e., we define $U:=U_{k}, u:=u_{k}$ and $e:=e_{k}$. We obtain

$$
\begin{aligned}
& \left\langle\nabla\left(\nabla \cdot\left(\frac{\nabla U_{k}}{\sqrt{\left|\nabla U_{k}\right|^{2}+\delta^{2}}}\right)-\nabla \cdot\left(\frac{\nabla u_{k}}{\sqrt{\left|\nabla u_{k}\right|^{2}+\delta^{2}}}\right)\right), \nabla \Delta e_{k+1}\right\rangle_{2} \\
= & \left\langle H_{1}(\nabla U) \cdot \nabla \Delta U-H_{1}(\nabla u) \cdot \nabla \Delta u, \nabla \Delta e_{k+1}\right\rangle_{2} \\
& +\left\langle H_{2}\left(U_{x}, U_{y}, U_{x x}, U_{x y}, U_{y y}\right) \cdot \nabla U_{x}-H_{2}\left(u_{x}, u_{y}, u_{x x}, u_{x y}, u_{y y}\right) \cdot \nabla u_{x}, \nabla \Delta e_{k+1}\right\rangle_{2} \\
& +\left\langle H_{3}\left(U_{x}, U_{y}, U_{x x}, U_{x y}, U_{y y}\right) \cdot \nabla U_{y}-H_{3}\left(u_{x}, u_{y}, u_{x x}, u_{x y}, u_{y y}\right) \cdot \nabla u_{y}, \nabla \Delta e_{k+1}\right\rangle_{2} \\
& +\left\langle H_{4}\left(U_{x}, U_{y}\right) \cdot \nabla U_{x x}-H_{4}\left(u_{x}, u_{y}\right) \cdot \nabla u_{x x}, \nabla \Delta e_{k+1}\right\rangle_{2} \\
& +\left\langle H_{5}\left(U_{x}, U_{y}\right) \cdot \nabla U_{x y}-H_{5}\left(u_{x}, u_{y}\right) \cdot \nabla u_{x y}, \nabla \Delta e_{k+1}\right\rangle_{2} \\
& +\left\langle H_{6}\left(U_{x}, U_{y}\right) \cdot \nabla U_{y y}-H_{6}\left(u_{x}, u_{y}\right) \cdot \nabla u_{y y}, \nabla \Delta e_{k+1}\right\rangle_{2}
\end{aligned}
$$




$$
\begin{aligned}
\leq & \frac{1}{2 \bar{\delta}}\left\|H_{1}(\nabla U) \cdot(\nabla \Delta U-\nabla \Delta u)\right\|_{2}^{2}+\frac{1}{2 \bar{\delta}}\left\|\nabla \Delta u \cdot\left(H_{1}(\nabla u)-H_{1}(\nabla U)\right)\right\|_{2}^{2} \\
& +\frac{1}{2 \bar{\delta}}\left\|H_{2}\left(U_{x}, U_{y}, U_{x x}, U_{x y}, U_{y y}\right) \cdot\left(\nabla U_{x}-\nabla u_{x}\right)\right\|_{2}^{2} \\
& +\frac{1}{2 \bar{\delta}}\left\|\nabla u_{x} \cdot\left(H_{2}\left(u_{x}, u_{y}, u_{x x}, u_{x y}, u_{y y}\right)-H_{2}\left(U_{x}, U_{y}, U_{x x}, U_{x y}, U_{y y}\right)\right)\right\|_{2}^{2} \\
& +\frac{1}{2 \bar{\delta}}\left\|H_{3}\left(U_{x}, U_{y}, U_{x x}, U_{x y}, U_{y y}\right) \cdot\left(\nabla U_{y}-\nabla u_{y}\right)\right\|_{2}^{2} \\
& +\frac{1}{2 \bar{\delta}}\left\|\nabla u_{y} \cdot\left(H_{3}\left(u_{x}, u_{y}, u_{x x}, u_{x y}, u_{y y}\right)-H_{3}\left(U_{x}, U_{y}, U_{x x}, U_{x y}, U_{y y}\right)\right)\right\|_{2}^{2} \\
& +\frac{1}{2 \bar{\delta}}\left\|H_{4}\left(U_{x}, U_{y}\right) \cdot\left(\nabla U_{x x}-\nabla u_{x x}\right)\right\|_{2}^{2}+\frac{1}{2 \bar{\delta}}\left\|\nabla u_{x x} \cdot\left(H_{4}\left(u_{x}, u_{y}\right)-H_{4}\left(U_{x}, U_{y}\right)\right)\right\|_{2}^{2} \\
& +\frac{1}{2 \bar{\delta}}\left\|H_{5}\left(U_{x}, U_{y}\right) \cdot\left(\nabla U_{x y}-\nabla u_{x y}\right)\right\|_{2}^{2}+\frac{1}{2 \bar{\delta}}\left\|\nabla u_{x y} \cdot\left(H_{5}\left(u_{x}, u_{y}\right)-H_{5}\left(U_{x}, U_{y}\right)\right)\right\|_{2}^{2} \\
& +\frac{1}{2 \bar{\delta}}\left\|H_{6}\left(U_{x}, U_{y}\right) \cdot\left(\nabla U_{y y}-\nabla u_{y y}\right)\right\|_{2}^{2}+\frac{1}{2 \bar{\delta}}\left\|\nabla u_{y y} \cdot\left(H_{6}\left(u_{x}, u_{y}\right)-H_{6}\left(U_{x}, U_{y}\right)\right)\right\|_{2}^{2} \\
& +6 \bar{\delta}\left\|\nabla \Delta e_{k+1}\right\|_{2}^{2},
\end{aligned}
$$

for a suitable constant $\bar{\delta}>0$. Next we want to use that the $H_{i}$ 's are Lipschitz continuous in $\Omega$, with Lipschitz constants $L(1 / \delta)<\infty$, for $\delta>0$, which grow as $\delta$ decreases. For simplicity, we only present the proof for the first part of $H_{2}$, i.e., for

$$
H_{2}^{1}\left(u_{x}, u_{y}, u_{x x}, u_{x y}, u_{y y}\right)=-\frac{\Delta u u_{x}+2\left(u_{x} u_{x x}+u_{y} u_{x y}\right)}{\left(|\nabla u|^{2}+\delta^{2}\right)^{3 / 2}}=\frac{u_{x}\left(3 u_{x x}+u_{y y}\right)+2 u_{y} u_{x y}}{\left(|\nabla u|^{2}+\delta^{2}\right)^{3 / 2}} .
$$

The others follow similarily. We have

$$
\begin{aligned}
& \left\|H_{2}^{1}\left(u_{x}, u_{y}, u_{x x}, u_{x y}, u_{y y}\right)-H_{2}^{1}\left(U_{x}, U_{y}, U_{x x}, U_{x y}, U_{y y}\right)\right\|_{2} \\
= & \left\|\frac{u_{x}\left(3 u_{x x}+u_{y y}\right)+2 u_{y} u_{x y}}{\left(|\nabla u|^{2}+\delta^{2}\right)^{3 / 2}}-\frac{U_{x}\left(3 U_{x x}+U_{y y}\right)+2 U_{y} U_{x y}}{\left(|\nabla U|^{2}+\delta^{2}\right)^{3 / 2}}\right\|_{2} \\
\leq & \left\|\frac{u_{x}\left(3 u_{x x}+u_{y y}\right)}{\left(|\nabla u|^{2}+\delta^{2}\right)^{3 / 2}}-\frac{U_{x}\left(3 U_{x x}+U_{y y}\right)}{\left(|\nabla U|^{2}+\delta^{2}\right)^{3 / 2}}\right\|_{2}+2\left\|\frac{u_{y} u_{x y}}{\left(|\nabla u|^{2}+\delta^{2}\right)^{3 / 2}}-\frac{U_{y} U_{x y}}{\left(|\nabla U|^{2}+\delta^{2}\right)^{3 / 2}}\right\|_{2} \\
\leq & \left\|\left(3 u_{x x}+u_{y y}\right)\left(\frac{u_{x}}{\left(|\nabla u|^{2}+\delta^{2}\right)^{3 / 2}}-\frac{U_{x}}{\left(|\nabla U|^{2}+\delta^{2}\right)^{3 / 2}}\right)\right\|_{2}+\left\|\frac{U_{x}}{\left(|\nabla U|^{2}+\delta^{2}\right)^{3 / 2}}\left(3 e_{x x}-e_{y y}\right)\right\|_{2} \\
& +2\left\|u_{x y}\left(\frac{u_{y}}{\left(|\nabla u|^{2}+\delta^{2}\right)^{3 / 2}}-\frac{U_{y}}{\left(|\nabla U|^{2}+\delta^{2}\right)^{3 / 2}}\right)\right\|_{2}+2\left\|\frac{U_{y}}{\left(|\nabla U|^{2}+\delta^{2}\right)^{3 / 2}} e_{x y}\right\|_{2} .
\end{aligned}
$$

From our assumption in Theorem 4.1 (iii) we have a continuous in time smooth solution $u$ on a finite time interval. In particular this gives us a uniform bound for the second derivatives of the exact solution $u$, i.e., there exists a $C>0$ such that $\left\|u_{x x}\right\|_{\infty}+\left\|u_{x y}\right\|_{\infty}+\left\|u_{y y}\right\|_{\infty}<C$ on a finite time interval $[0, T]$. Further, with the fact that the function $\frac{x}{\left(x^{2}+y^{2}+\delta^{2}\right)^{3 / 2}}$ is uniformly bounded for $\delta>0$ and for all $x, y \in \mathbb{R}$ we have

$$
\begin{aligned}
& \quad\left\|H_{2}^{1}\left(u_{x}, u_{y}, u_{x x}, u_{x y}, u_{y y}\right)-H_{2}^{1}\left(U_{x}, U_{y}, U_{x x}, U_{x y}, U_{y y}\right)\right\|_{2} \\
& \leq C\left\|\frac{u_{x}}{\left(|\nabla u|^{2}+\delta^{2}\right)^{3 / 2}}-\frac{U_{x}}{\left(|\nabla U|^{2}+\delta^{2}\right)^{3 / 2}}\right\|_{2}+C\left\|3 e_{x x}-e_{y y}\right\|_{2} \\
& +2 C\left\|\frac{u_{y}}{\left(|\nabla u|^{2}+\delta^{2}\right)^{3 / 2}}-\frac{U_{y}}{\left(|\nabla U|^{2}+\delta^{2}\right)^{3 / 2}}\right\|_{2}+2 C\left\|e_{x y}\right\|_{2},
\end{aligned}
$$


where we used a universal constant $C>0$ for the uniform bounds. Moreover, for a fixed $y$ and $\delta>0$ the function $\frac{x}{\left(x^{2}+y^{2}+\delta^{2}\right)^{3 / 2}}$ is Lipschitz continuous with constant $L(1 / \delta)$, which is increasing as $\delta$ decreases. By additionally applying the triangular inequality once more we eventually have

$$
\begin{aligned}
& \left\|H_{2}^{1}\left(u_{x}, u_{y}, u_{x x}, u_{x y}, u_{y y}\right)-H_{2}^{1}\left(U_{x}, U_{y}, U_{x x}, U_{x y}, U_{y y}\right)\right\|_{2} \\
\leq & C L(1 / \delta)\left(\left\|e_{x}\right\|_{2}+\left\|e_{y}\right\|_{2}+\|\nabla e\|_{2}\right)+C\left(\left\|e_{x x}\right\|_{2}+\left\|e_{x y}\right\|_{2}+\left\|e_{y y}\right\|_{2}\right),
\end{aligned}
$$

and hence that $H_{2}^{1}$ is Lipschitz continuous. Similarly one can show that the other $H_{i}$ 's are Lipschitz continuous. Let us further observe that $H_{1}, H_{4}, H_{5}, H_{6}$ are uniformly bounded for $\delta>0$. Moreover, the uniform boundedness of $H_{2}$ and $H_{3}$ for the discrete in time solution $U$ on a finite time interval is given by the smoothness assumption in Theorem 4.1 (iii) for $U$. Then, with the Lipschitz continuity and the uniform boundedness of the $H_{i}$ 's on a finite time interval, and the uniform boundedness on a finite time interval of $\nabla u_{k}, \Delta u_{k}$, and $\nabla \Delta u_{k}$ for the exact solution $u_{k}$ given in Theorem 4.1 (iii), we eventually obtain an estimate for (4.10):

$$
\begin{gathered}
\left\langle\nabla\left(\nabla \cdot\left(\frac{\nabla U_{k}}{\sqrt{\left|\nabla U_{k}\right|^{2}+\delta^{2}}}\right)-\nabla \cdot\left(\frac{\nabla u_{k}}{\sqrt{\left|\nabla u_{k}\right|^{2}+\delta^{2}}}\right)\right), \nabla \Delta e_{k+1}\right\rangle_{2} \\
\leq \frac{C}{2 \bar{\delta}}\|\nabla \Delta e\|_{2}^{2}+C L \frac{3}{\bar{\delta}}\|\nabla e\|_{2}^{2}+\frac{C}{2 \bar{\delta}}\left\|\nabla e_{x}\right\|_{2}^{2}+\frac{C}{2 \bar{\delta}}\left\|\nabla e_{y}\right\|_{2}^{2}+\frac{C L}{\bar{\delta}}\left(\left\|e_{x x}\right\|_{2}^{2}+\left\|e_{x y}\right\|_{2}^{2}+\left\|e_{y y}\right\|_{2}^{2}\right) \\
+\frac{C}{2 \bar{\delta}}\left\|\nabla e_{x x}\right\|_{2}^{2}+\frac{C}{2 \bar{\delta}}\left\|\nabla e_{x y}\right\|_{2}^{2}+\frac{C}{2 \bar{\delta}}\left\|\nabla e_{y y}\right\|_{2}^{2}+6 \bar{\delta}\left\|\nabla \Delta e_{k+1}\right\|_{2}^{2}, \quad \text { (4.11) }
\end{gathered}
$$

where $L=L(1 / \delta)$ denotes a universal Lipschitz constant for the $H_{i}$ 's and $C$ is a universal constant for the involved uniform bounds.

Further, having assumed zero Neumann boundary conditions for (1.5) and (4.4), i.e.,

$$
\nabla u \cdot \vec{n}=\nabla \nabla \cdot\left(\frac{\nabla u}{\sqrt{|\nabla u|^{2}+\delta^{2}}}\right) \cdot \vec{n}=0, \quad \text { on } \partial \Omega,
$$

where $\vec{n}$ is the outward pointing normal on $\partial \Omega$, the second and third derivatives in (4.11) can be bounded by

$$
\left\|e_{x x}\right\|_{2}^{2}+\left\|e_{x y}\right\|_{2}^{2}+\left\|e_{y y}\right\|_{2}^{2}+\left\|\nabla e_{x x}\right\|_{2}^{2}+\left\|\nabla e_{x y}\right\|_{2}^{2}+\left\|\nabla e_{y y}\right\|_{2}^{2} \leq B\left(\|\Delta e\|_{2}^{2}+\|\nabla \Delta e\|_{2}^{2}\right),
$$

for a suitable constant $B>0$. Because of the Neumann boundary conditions we also get that $\int_{\Omega} \Delta e=0$. Hence, we can apply Poincaré's inequality to $\|\Delta e\|_{2}$ and obtain, for (4.9),

$$
\begin{aligned}
& \left(\frac{1}{2 \Delta t}+C_{2}\left(1-\delta_{2}\right)-\delta_{3}\right)\left\|\nabla e_{k+1}\right\|_{2}^{2}+\left(C_{1}\left(1-\delta_{1}\right)-\delta_{4}-6 \bar{\delta}\right)\left\|\nabla \Delta e_{k+1}\right\|_{2}^{2} \\
\leq & \left(\frac{1}{2 \Delta t}+\frac{C_{2}}{\delta_{2}}+\frac{\lambda_{0}^{2}}{\delta_{3}} \frac{3 C L}{\bar{\delta}}\right)\left\|\nabla e_{k}\right\|_{2}^{2}+\left(\frac{C_{1}}{\delta_{1}}+\frac{C}{2 \bar{\delta}}+B C\left[\frac{5}{2 \bar{\delta}}+L \frac{1}{\bar{\delta}}\right]\right)\left\|\nabla \Delta e_{k}\right\|_{2}^{2}+\frac{1}{\delta_{4}}\left\|\tau_{k}\right\|_{-1}^{2},
\end{aligned}
$$

where we reintroduced the index notation $e_{k}$ for $e$. Therefore by following the lines of the proof of Proposition 3.4 we finally have, for $k \Delta t \leq T$,

$$
\left\|\nabla e_{k}\right\|_{2}^{2}+\Delta t M_{1}\left\|\nabla \Delta e_{k}\right\|_{2}^{2} \leq \frac{T}{M_{2}} e^{M_{3} T}(\Delta t)^{2},
$$


for suitable positive constants $M_{1}, M_{2}$ and $M_{3}$.

REMARK 4.5. Note that the Lipschitz continuity of the $H_{i}$ 's - necessary for the estimates in the convergence proof - breaks down if $\delta \rightarrow 0$, where $\delta$ is the smoothing parameter in the square-root regularization (4.3) of the total variation.

4.2. Numerical results. Numerical results for the $\mathrm{TV}-\mathrm{H}^{-1}$ inpainting approach are presented in Figures 4.1 and 4.2. For a comparison of the higher order $\mathrm{TV}-\mathrm{H}^{-1}$ inpainting approach with its second order cousin, the standard TV-L $\mathrm{L}^{2}$ inpainting method, in Figure 4.2 we consider the performance of both algorithms in a small part of the image in Figure 4.1. In fact the result shown in Figures 4.1 and 4.2 strongly indicates the continuation of the gradient of the image function into the inpainting domain. A rigorous proof of this observation, as the one for Cahn-Hilliard inpainting (cf. Section 3), is a matter of future research. In both examples the total variation $|\nabla u|$ is approximated by $\sqrt{|\nabla u|^{2}+\delta}$ and the time step size $\Delta t$ is chosen to be equal to one. The computational time for the example in Figure 4.1 is of the order of 100 seconds on a $1.86 \mathrm{GHz}$ processor with $1 \mathrm{~GB}$ RAM.
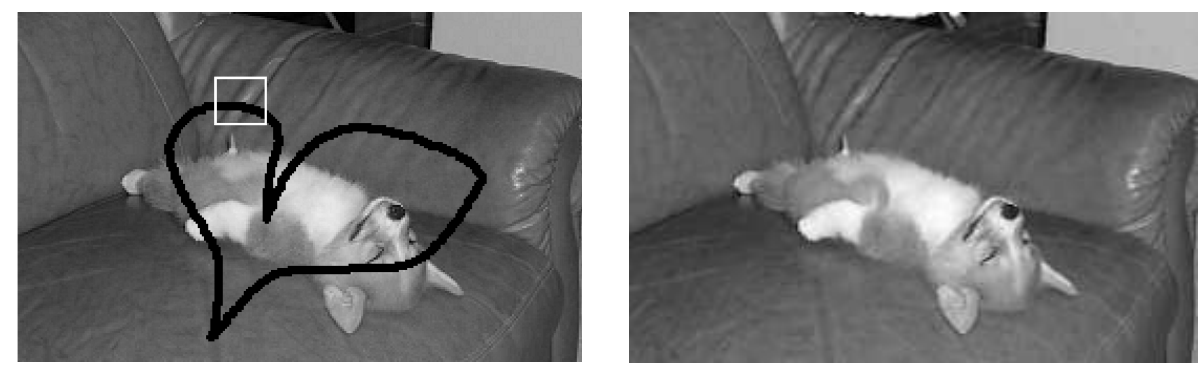

FIG. 4.1. $T V-H^{-1}$ inpainting: $u(1000)$ with $\lambda_{0}=10^{3}$
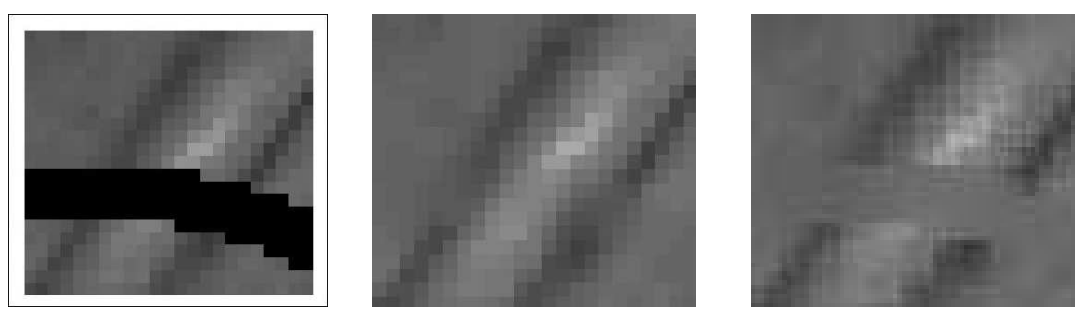

FIG. 4.2. (l.) $u(1000)$ with $T V-H^{-1}$ inpainting, (r.) $u(5000)$ with $T V$ - $L^{2}$ inpainting

\section{LCIS inpainting}

Our last example for the applicability of the convexity splitting method to higherorder inpainting approaches is inpainting with LCIS (1.6). With $f \in L^{2}(\Omega)$ our inpainted image $u$ evolves in time as

$$
u_{t}=-\Delta(\arctan (\Delta u))+\lambda(f-u) .
$$

In contrast to the other two inpainting methods that we discussed, this inpainting 
equation is a gradient flow in $L^{2}$ for the energy

$$
E(u)=\int_{\Omega} G(\Delta u) d x+\frac{1}{2} \int_{\Omega} \lambda(f-u)^{2},
$$

with $G^{\prime}(y)=\arctan (y)$. Therefore Eyre's result in Theorem 2.3 can be applied directly. The functional $E(u)$ is split into $E_{c}-E_{e}$ with

$$
\begin{aligned}
& E_{c}(u)=\int_{\Omega} \frac{C_{1}}{2}(\Delta u)^{2} d x+\frac{1}{2} \int_{\Omega} \frac{C_{2}}{2}|u|^{2} d x \\
& E_{e}(u)=\int_{\Omega}-G(\Delta u)+\frac{C_{1}}{2}(\Delta u)^{2} d x+\frac{1}{2} \int_{\Omega}-\lambda(f-u)^{2}+\frac{C_{2}}{2}|u|^{2} d x .
\end{aligned}
$$

The resulting time-stepping scheme is

$$
\frac{U_{k+1}-U_{k}}{\Delta t}+C_{1} \Delta^{2} U_{k+1}+C_{2} U_{k+1}=-\Delta\left(\arctan \left(\Delta U_{k}\right)\right)+C_{1} \Delta^{2} U_{k}+\lambda\left(f-U_{k}\right)+C_{2} U_{k} .
$$

Again we impose homogeneous Neumann boundary conditions, use DCT to solve (5.1), and choose the constants $C_{1}$ and $C_{2}$ such that $E_{c}$ and $E_{e}$ are all strictly convex and condition (2.7) is satisfied. The functional $E_{c}$ is convex for all $C_{1}, C_{2}>0$. The first term in $E_{e}$ is convex if $C_{1}>1$. This follows from its second variation, namely

$$
\begin{aligned}
\nabla^{2} E_{1 e}(u)(v, w) & =\left(\frac{d}{d s} \int\left(C_{1} \Delta(u+s w)-\arctan (\Delta(u+s w))\right) \Delta v d x\right)_{s=0} \\
& =\int\left(C_{1}-\frac{1}{1+(\Delta u)^{2}}\right) \Delta v \Delta w d x
\end{aligned}
$$

For $E_{1 e}$ to be convex, $\nabla^{2} E_{1 e}(u)(v, w)$ must be $>0$ for all $v, w \in C^{\infty}$, and therefore

$$
C_{1}-\frac{1}{1+(\Delta u)^{2}}>0
$$

Substituting $s=\Delta u$ we obtain

$$
C_{1}>\frac{1}{1+s^{2}} \quad \forall s \in \mathbb{R}
$$

This inequality is fulfilled for all $s \in \mathbb{R}$ if $C_{1}>1$. We obtain the same condition on $C_{1}$ for $G^{\prime}(s)=\arctan \left(\frac{s}{\delta}\right)$. For the convexity of the second term of $E_{e}$, the second constant has to fulfill $C_{2}>\lambda_{0}$; cf. the computation for the fitting term in Section 4 . With these choices of $C_{1}$ and $C_{2}$ also condition (2.7) of Theorem 2.3 is automatically satisfied.

5.1. Rigorous estimates for the scheme. Finally we present rigorous results for (5.1). In contrast to the inpainting Equations (1.4) and (1.5), inpainting with LCIS follows a variational principle. Hence, by choosing the constants $C_{1}$ and $C_{2}$ appropriately, i.e., $C_{1}>1, C_{2}>\lambda_{0}$ (cf. the computations above), Theorem 2.3 ensures that the iterative scheme (5.1) is unconditionally gradient stable. In addition to this property, we present similar results as before for Cahn-Hilliard and $\mathrm{TV}-\mathrm{H}^{-1}$ inpainting.

THEOREM 5.1. Let $u$ be the exact solution of (1.6) and $u_{k}=u(k \Delta t)$ the exact solution at time $k \Delta t$, for a time step $\Delta t>0$ and $k \in \mathbb{N}$. Let $U_{k}$ be the kth iterate of (5.1) with constants $C_{1}>1, C_{2}>\lambda_{0}$. Then the following statements hold: 
(i) Under the assumption that $\left\|u_{t t}\right\|_{-1}$ and $\left\|\nabla \Delta u_{t}\right\|_{2}$ are bounded, the numerical scheme (5.1) is consistent with the continuous Equation (1.6) and of order one in time.

(ii) The solution sequence $U_{k}$ is bounded on a finite time interval $[0, T]$ for all $\Delta t>0$. In particular, for $k \Delta t<T, T>0$ fixed, we have

$$
\left\|\nabla U_{k}\right\|_{2}^{2}+\Delta t K_{1}\left\|\nabla \Delta U_{k}\right\|_{2}^{2} \leq e^{K_{2} T}\left(\left\|\nabla U_{0}\right\|_{2}^{2}+\Delta t K_{1}\left\|\nabla \Delta U_{0}\right\|_{2}^{2}+\Delta t T C\left(\Omega, D, \lambda_{0}, f\right)\right)
$$

for suitable constants $K_{1}, K_{2}$, and a constant $C$ depending on $\Omega, D, \lambda_{0}, f$ only.

(iii) Let $e_{k}=u_{k}-U_{k}$. If

$$
\left\|\nabla \Delta u_{k}\right\|_{2}^{2} \leq K, \text { for a constant } K>0, \text { and for all } k \Delta t<T
$$

then the error $e_{k}$ converges to zero as $\Delta t \rightarrow 0$. In particular, for $k \Delta t \leq T$, $T>0$ fixed, we have

$$
\left\|\nabla e_{k}\right\|_{2}^{2}+\Delta t M_{1}\left\|\nabla \Delta e_{k}\right\|_{2}^{2} \leq \frac{T}{M_{2}} e^{M_{3} T}(\Delta t)^{2}
$$

for suitable nonnegative constants $M_{1}, M_{2}$ and $M_{3}$.

REMARK 5.1. As in Theorem 4.1 (cf. also Remark 4.1) the convergence of the iterates $U_{k}$ to the exact solution is proven under an assumption on the exact solution, i.e., assumption (5.3), whose validity is unknown in general. However, previous results in [15] for the denoising case, i.e., for $\lambda(x)=\lambda_{0}$ in all of $\Omega$, and for smooth initial data and smooth $f$, suggest the assumption is also reasonable for the inpainting case.

The proof of Theorem 5.1 is organized in the following three Propositions 5.2-5.4. Since the proof of consistency follows the lines of Proposition 3.2 and Proposition 4.2, we just state the result.

Proposition 5.2 (Consistency (i)). Under the same assumptions as in Theorem 5.1 and in particular assuming that $\left\|u_{t t}\right\|_{-1}$ and $\left\|\nabla \Delta u_{t}\right\|_{2}$ are bounded, we have

$$
\left\|\tau_{k}\right\|_{-1}=O(\Delta t) \quad \text { for } \Delta t \rightarrow 0
$$

Next we would like to show the boundedness of a solution of (5.1) in the following proposition.

Proposition 5.3. (Unconditional stability (ii)) Under the same assumptions as in Theorem 5.1 the solution sequence $U_{k}$ fulfills (5.2). This gives boundedness of the solution sequence on $[0, T]$.

Proof. If we multiply (5.1) with $-\Delta U_{k+1}$ and integrate over $\Omega$, we obtain

$$
\begin{aligned}
\frac{1}{\Delta t}\left(\left\|\nabla U_{k+1}\right\|_{2}^{2}-\left\langle\nabla U_{k}, \nabla U_{k+1}\right\rangle_{2}\right) & +C_{2}\left\|\nabla U_{k+1}\right\|_{2}^{2}+C_{1}\left\|\nabla \Delta U_{k+1}\right\|_{2}^{2} \\
=\left\langle\Delta \arctan \left(\Delta U_{k}\right)\right. & \left., \Delta U_{k+1}\right\rangle_{2}+C_{1}\left\langle\nabla \Delta U_{k}, \nabla \Delta U_{k+1}\right\rangle_{2} \\
& +\left\langle\nabla\left(\lambda\left(f-U_{k}\right)\right), \nabla U_{k+1}\right\rangle_{2}+C_{2}\left\langle\nabla U_{k}, \nabla U_{k+1}\right\rangle_{2} .
\end{aligned}
$$


Using the same arguments as in the proofs of Proposition 3.3 and 4.3 we obtain

$$
\begin{aligned}
& \frac{1}{2 \Delta t}\left(\left\|\nabla U_{k+1}\right\|_{2}^{2}-\left\|\nabla U_{k}\right\|_{2}^{2}\right)+C_{2}\left\|\nabla U_{k+1}\right\|_{2}^{2}+C_{1}\left\|\nabla \Delta U_{k+1}\right\|_{2}^{2} \\
& \leq\left\langle\Delta \arctan \left(\Delta U_{k}\right), \Delta U_{k+1}\right\rangle_{2}+\frac{C_{1}}{\delta_{1}}\left\|\nabla \Delta U_{k}\right\|_{2}^{2}+C_{1} \delta_{1}\left\|\nabla \Delta U_{k+1}\right\|_{2}^{2}+\frac{\lambda_{0}^{2}}{2 \delta_{2}}\left\|\nabla U_{k}\right\|_{2}^{2} \\
& \quad+\delta_{2}\left\|\nabla U_{k+1}\right\|_{2}^{2}+\frac{C_{2}}{\delta_{3}}\left\|\nabla U_{k}\right\|_{2}^{2}+C_{2} \delta_{3}\left\|\nabla U_{k+1}\right\|_{2}^{2}+C\left(\Omega, D, \lambda_{0}, f\right) .
\end{aligned}
$$

Now, the first term on the right side of the inequality can be estimated as follows

$$
\begin{aligned}
\left\langle\Delta \arctan \left(\Delta U_{k}\right), \Delta U_{k+1}\right\rangle_{2} & =-\left\langle\nabla \arctan \left(\Delta U_{k}\right), \nabla \Delta U_{k+1}\right\rangle_{2} \\
& =-\left\langle\frac{1}{1+\left(\Delta U_{k}\right)^{2}} \nabla \Delta U_{k}, \nabla \Delta U_{k+1}\right\rangle_{2} \\
& \leq \frac{1}{\delta_{4}}\left\|\frac{1}{1+\left(\Delta U_{k}\right)^{2}} \nabla \Delta U_{k}\right\|_{2}^{2}+\delta_{4}\left\|\nabla \Delta U_{k+1}\right\|_{2}^{2} \\
& \leq \frac{1}{\delta_{4}}\left\|\nabla \Delta U_{k}\right\|_{2}^{2}+\delta_{4}\left\|\nabla \Delta U_{k+1}\right\|_{2}^{2} .
\end{aligned}
$$

From this we get

$$
\begin{aligned}
& \left(\frac{1}{2 \Delta t}+C_{2}\left(1-\delta_{3}\right)-\delta_{2}\right)\left\|\nabla U_{k+1}\right\|_{2}^{2}+\left(C_{1}\left(1-\delta_{1}\right)-\delta_{4}\right)\left\|\nabla \Delta U_{k+1}\right\|_{2}^{2} \\
\leq & \left(\frac{1}{2 \Delta t}+\frac{\lambda_{0}^{2}}{2 \delta_{2}}+\frac{C_{2}}{\delta_{3}}\right)\left\|\nabla U_{k}\right\|_{2}^{2}+\left(\frac{C_{1}}{\delta_{1}}+\frac{1}{\delta_{4}}\right)\left\|\nabla \Delta U_{k}\right\|_{2}^{2}+C\left(\Omega, D, \lambda_{0}, f\right) .
\end{aligned}
$$

Analogously to Section 4.1, with

$$
C_{a}=1+\Delta t\left(C_{2}-1\right), \quad C_{b}=C_{1}-1, \quad C_{c}=1+2 \Delta t\left(\lambda_{0}^{2}+2 C_{2}\right), \quad C_{d}=4\left(C_{1}+1\right),
$$

we obtain

$$
\left\|\nabla U_{k}\right\|_{2}^{2}+\Delta t \frac{C_{b}}{C_{a}}\left\|\nabla \Delta U_{k}\right\|_{2}^{2} \leq e^{K T}\left(\left\|\nabla U_{0}\right\|_{2}^{2}+\Delta t \frac{C_{b}}{C_{a}}\left\|\nabla \Delta U_{0}\right\|_{2}^{2}+\Delta t T \frac{2}{C_{a}} C\left(\Omega, D, \lambda_{0}, f\right)\right),
$$

which gives boundedness of the solution sequence on $[0, T]$ for any $T>0$ and any $\Delta t>0$.

The convergence of the discrete solution to the continuous one as $\Delta t \rightarrow 0$ is verified in the following proposition.

Proposition 5.4 (Convergence (iii)). Under the same assumptions as in Theorem 5.1 and in particular under assumption (5.3), the error $e_{k}$ fulfills (5.4).

Proof. Since all the computations in the convergence proof for (5.1) are the same as in Section 4.1 for (4.4) except of the estimate for the regularizer $\Delta(\arctan (\Delta u))$, we only give the details for the latter and leave the rest to the reader. Thus, for the inner product involving the regularizer of (5.1) within the convergence proof, we obtain

$$
\begin{aligned}
& \left\langle-\Delta\left(\arctan \left(\Delta U_{k}\right)-\arctan \left(\Delta u_{k}\right)\right), \Delta e_{k+1}\right\rangle_{2} \\
= & \left\langle\nabla\left(\arctan \left(\Delta U_{k}\right)-\arctan \left(\Delta u_{k}\right)\right), \nabla \Delta e_{k+1}\right\rangle_{2} \\
= & \left\langle w\left(\Delta U_{k}\right) \nabla \Delta U_{k}-w\left(\Delta u_{k}\right) \nabla \Delta u_{k}, \nabla \Delta e_{k+1}\right\rangle_{2} \\
= & -\left\langle w\left(\Delta U_{k}\right) \nabla \Delta e_{k}, \nabla \Delta e_{k+1}\right\rangle_{2}-\left\langle\left(w\left(\Delta U_{k}\right)-w\left(\Delta u_{k}\right)\right) \nabla \Delta u_{k}, \nabla \Delta e_{k+1}\right\rangle_{2} \\
\leq & \frac{1}{2 \delta}\left\|w\left(\Delta U_{k}\right)\left|\nabla \Delta e_{k}\right|\right\|_{2}^{2}+\frac{1}{2 \delta_{1}}\left\|\left(w\left(\Delta u_{k}\right)-w\left(\Delta U_{k}\right)\right)\left|\nabla \Delta u_{k}\right|\right\|_{2}^{2}+\frac{\delta+\delta_{1}}{2}\left\|\nabla \Delta e_{k+1}\right\|_{2}^{2},
\end{aligned}
$$


where we have used that

$$
\nabla(\arctan (\Delta u))=\frac{1}{1+|\Delta u|^{2}} \nabla \Delta u=w(\Delta u) \nabla \Delta u
$$

Using the uniform boundedness of $w(s)$ for all $s \in \mathbb{R}$, the uniform bound on $\nabla \Delta u_{k}$ from Assumption (5.3), and the Lipschitz continuity of $w$, we get

$$
\begin{gathered}
\left\langle-\Delta\left(\arctan \left(\Delta U_{k}\right)-\arctan \left(\Delta u_{k}\right)\right), \Delta e_{k+1}\right\rangle_{2} \\
\leq \frac{C}{2 \delta}\left\|\nabla \Delta e_{k}\right\|_{2}^{2}+\frac{C L}{2 \delta_{1}}\left\|\Delta e_{k}\right\|_{2}^{2}+\frac{\delta+\delta_{1}}{2}\left\|\nabla \Delta e_{k+1}\right\|_{2}^{2} .
\end{gathered}
$$

Moreover, because of the zero Neumann boundary conditions fulfilled by solutions of (1.6) and (5.1), i.e.,

$$
\nabla u \cdot \vec{n}=\nabla(\arctan (\Delta u)) \cdot \vec{n}=0, \quad \text { on } \partial \Omega,
$$

where $\vec{n}$ is the outward pointing normal on $\partial \Omega, \Delta e_{k}$ has zero mean and we can apply Poincaré's inequality to obtain

$$
\begin{aligned}
& \left\langle-\Delta\left(\arctan \left(\Delta U_{k}\right)-\arctan \left(\Delta u_{k}\right)\right), \Delta e_{k+1}\right\rangle_{2} \\
\leq & \left(\frac{C}{2 \delta}+\frac{C L}{2 \delta_{1}}\right)\left\|\nabla \Delta e_{k}\right\|_{2}^{2}+\frac{\delta+\delta_{1}}{2}\left\|\nabla \Delta e_{k+1}\right\|_{2}^{2} .
\end{aligned}
$$

Following the same steps as in the proof of Proposition 4.4 we finally have, for $k \Delta t \leq T$,

$$
\left\|\nabla e_{k}\right\|_{2}^{2}+\Delta t M_{1}\left\|\nabla \Delta e_{k}\right\|_{2}^{2} \leq \frac{T}{M_{2}} e^{M_{3} T}(\Delta t)^{2}
$$

for suitable positive constants $M_{1}, M_{2}$ and $M_{3}$.

5.2. Numerical results. For the comparison with $\mathrm{TV}-\mathrm{H}^{-1}$ inpainting we apply (5.1) to the same image as in Section 4.2. This example is presented in Figure 5.1. In Figure 5.2 the LCIS inpainting result is compared with $\mathrm{TV}-\mathrm{H}^{-1}$ - and TV-L $\mathrm{L}^{2}$ inpainting, for a small part in the given image. Again the result of this comparison indicates the continuation of the gradient of the image function into the inpainting domain for the two higher-order methods. A rigorous proof of this observation is a matter of future research. For the numerical computation of (5.1) the $\arctan (s)$ was regularized by $\arctan (s / \delta), \delta>0$ and $\Delta t$ chosen to be equal to 0.01 . The inpainted image in Figure 5.1 has been computed in about 90 seconds on a $1.86 \mathrm{GHz}$ processor with 1 GB RAM.

\section{Conclusion}

In this paper we present several higher order PDE-based methods for image inpainting, along with unconditionally stable time-stepping schemes for the solution of these equations. Specific examples discussed include Cahn-Hilliard inpainting, TV$\mathrm{H}^{-1}$ inpainting, and inpainting with LCIS. The construction of these schemes is based on the idea of convexity splitting, also introduced in this paper. We study the numerical analysis of the schemes including consistency, unconditional stability, and convergence. Below we consider some open problems for this class of methods. 

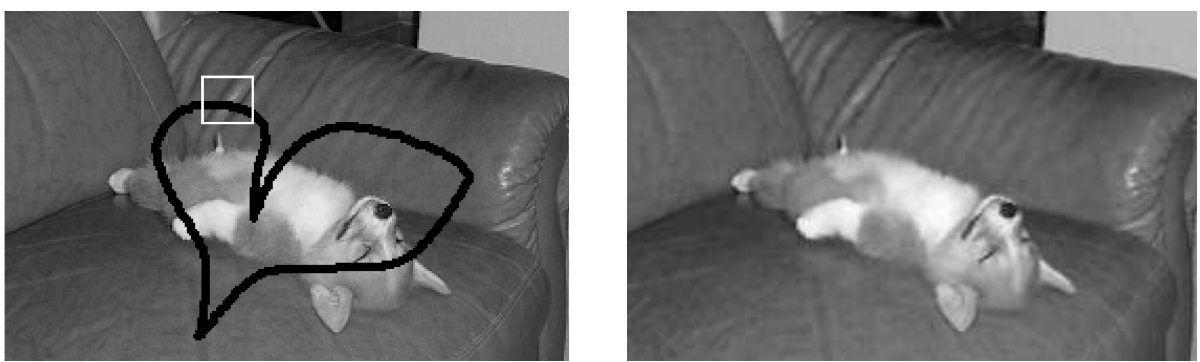

FIG. 5.1. LCIS inpainting $u(500)$ with $\delta=0.1$ and $\lambda_{0}=10^{2}$.
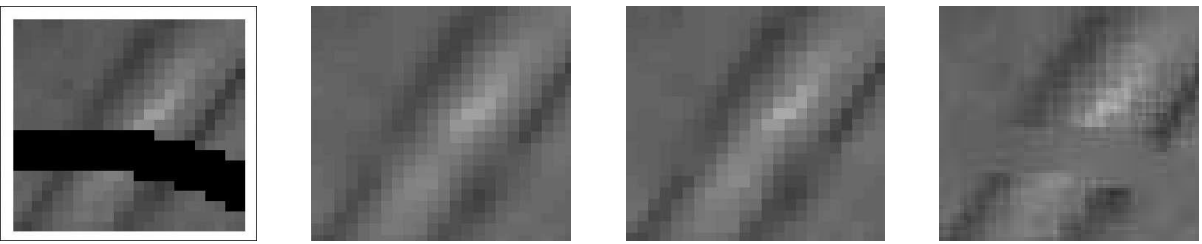

FIG. 5.2. (l.) $u(1000)$ with LCIS inpainting, (m.) $u(1000)$ with $T V-H^{-1}$ inpainting, (r.) $u(5000)$ with $T V-L^{2}$ inpainting

- The advantage of fourth order inpainting models, over models of second differential order, is the smooth continuation of image contents, including direction of edges, across gaps in the image. Fourth order PDEs require an extra boundary condition compared with second order equations and this is the motivation for additional geometric content provided by such methods. However, in general, the additional boundary condition could involve any of the higher derivatives, and for inpainting is it desirable to continue the first derivative accross the inpainting region. The methods proposed here are global methods based on an $L^{2}$ fidelity term associated with the known information. For the special case of the Cahn-Hilliard equation [14], in the limit as $\lambda_{0} \rightarrow \infty$ a stationary solution is proved to satisfy precisely the desired two boundary conditions - matching of grey value and matching direction of edges. We conjecture that analogous results are true for the other methods presented here although a rigorous proof is beyond the scope of this manuscript.

- For the proofs of convergence of the discrete solution to the exact solution, i.e., for the proofs of Theorem 4.4 and Theorem 5.4, we had to assume that the exact solution is bounded on a finite time interval in a certain Sobolev norm. As we already argued in the remarks after the statement of the theorems, these assumptions seem to be heuristically reasonable considering earlier results in $[15,16]$. Nevertheless a rigorous derivation of such bounds is still missing.

- Besides the fact that rigorous results for fourth-order partial differential equations are rare in general, an asymptotic analysis of our three inpainting models would be of high (even practical) interest. More precisely the convergence of a solution of the evolution Equations (1.4), (1.5), and (1.6), to a stationary state is still open. Since the inpainted image is the stationary solution 
of those evolution equations, the asymptotic behavior is of course an issue. Also, in practice the numerical schemes are solved to steady state (up to an approximational error). Note that in addition to the fourth differential order, a difficulty in the convergence analysis of (1.4) and (1.5) is that the equations do not follow a variational principle.

- The discrete schemes proposed in this paper are unconditionally stable and their numerical performance is a matter of 10 to 100 seconds for small to medium-sized images, i.e., $128 \times 128$ to $256 \times 256$ pixels, and gaps that constitute about one to ten percent of the image domain. Fast numerical solvers for higher order inpainting models is still a mostly open field of research. Among such fast solvers we found the recent contribution of Brito-Loeza and Chen [18] very interesting and forward-looking, who use a multigrid method to solve inpainting with CDD (Curvature Driven Diffusion). Another approach is the Split Bregman method of Goldstein and Osher [41, 42], which suggests a splitting of a higher-order variational problem in two consecutively minimized first-order problems. Although not directly applicable to the nonvariational inpainting techniques (1.4) and (1.5), their method promises an efficient solution of, e.g., (1.6) LCIS inpainting.

Acknowledgments. C.-B. Schönlieb acknowledges the financial support provided by the DFG Graduiertenkolleg 1023 Identification in Mathematical Models: Synergy of Stochastic and Numerical Methods, support by the project WWTF Five senses-Call 2006, Mathematical Methods for Image Analysis and Processing in the Visual Arts and by the FFG project Erarbeitung neuer Algorithmen zum Image Inpainting, projectnumber 813610 . Further, this publication is based on work supported by Award No. KUK-I1-007-43, made by King Abdullah University of Science and Technology (KAUST). In addition, C.-B. Schönlieb thanks IPAM (Institute for Pure and Applied Mathematics), UCLA, for the hospitality and the financial support during the preparation of this work.

Both authors acknowledge support from the NSF grant BCS-0527388, ONR grant N000140810363, and the Department of Defense.

The authors thank the referees and Wenuah Gao (UCLA) for useful comments on the manuscript.

\section{REFERENCES}

[1] J.-F. Aujol and A. Chambolle, Dual norms and image decomposition models, International Journal of Computer Vision, 63(1), 85-104, June, 2005.

[2] J.-F. Aujol and G. Gilboa, Constrained and SNR-based solutions for TV-Hilbert space image denoising, J. Math. Imaging and Vision, 26(1-2), 217-237, November 2006.

[3] W. Baatz, M. Fornasier, P. Markowich and C.B. Schönlieb, Inpainting of ancient Austrian frescoes, Conference Proceedings of Bridges 2008, Leeuwarden 2008, 150-156, 2008.

[4] J.W. Barrett and J.F. Blowey, Finite element approximation of a model for phase separation of a multi-component alloy with non-smooth free energy, Numer. Math., 77(1), 1-34, 1997.

[5] J.W. Barrett and J.F. Blowey, Finite element approximation of a model for phase separation of a multi-component alloy with a concentration-dependent mobility matrix, IMA J. Numer. Anal., 18(2), 287-328, 1998.

[6] J.W. Barrett and J.F. Blowey, Finite element approximation of a model for phase separation of a multi-component alloy with nonsmooth free energy and a concentration dependent mobility matrix, Math. Models Methods Appl. Sci., 9(5), 627-663, 1999.

[7] J.W. Barrett and J.F. Blowey, Finite element approximation of the Cahn-Hilliard equation with concentration dependent mobility, Math. Comput., 68(226), 487-517, 1999. 
[8] J. Becker, G. Grün, M. Lenz and M. Rumpf, Numerical methods for fourth order nonlinear diffusion problems, Applications of Mathematics, 47, 517-543, 2002.

[9] J. Bect, L. Blanc-Féraud, G. Aubert and A. Chambolle, A $\ell^{1}$-unified Variational Framework for Image Restoration, T. Pajdla and J. Matas (eds.), Proceedings of the 8th European Conference on Computer Vision IV, Prague, Czech Republic, Spinger Verlag, 2004.

[10] M. Bertalmio, G. Sapiro, V. Caselles and C. Ballester, Image inpainting, Siggraph 2000, Computer Graphics Proceedings, 417-424, 2000.

[11] M. Bertalmio, A.L. Bertozzi and G. Sapiro, Navier-Stokes, fluid dynamics, and image and video inpainting, Proceedings of the 2001 IEEE Computer Society Conference on Computer Vision and Pattern Recognition, 1, 355-362, 2001.

[12] M. Bertalmio, L. Vese, G. Sapiro and S. Osher, Simultaneous structure and texture image inpainting, IEEE Trans. Image Proc., 12(8), 882-889, 2003.

[13] A. Bertozzi, S. Esedoglu and A. Gillette, Inpainting of binary images using the Cahn-Hilliard equation, IEEE Trans. Image Proc., 16(1), 285-291, 2007.

[14] A. Bertozzi, S. Esedoglu and A. Gillette, Analysis of a two-scale Cahn-Hilliard model for image inpainting, Multiscale Modeling and Simulation, 6(3), 913-936, 2007.

[15] A. Bertozzi and J.B. Greer, Low-curvature image simplifiers: global regularity of smooth solutions and Laplacian limiting schemes, Commun. Pure Appl. Math., LVII, 764-790, 2004.

[16] A. Bertozzi, J. Greer, S. Osher and K. Vixie, Nonlinear regularizations of TV based PDEs for image processing, AMS Series of Contemporary Mathematics, G.-Q. Chen, G. Gasper, and J. Jerome (eds.), 371, 29-40, 2005.

[17] A. Bertozzi, N. Ju and H.W. Lu, A biharmonic-modified forward time stepping method for fourth order nonlinear diffusion equations, Discrete and Continuous Dynamical Systems 2009, submitted. UCLA CAM reports 08-18, 2008.

[18] C. Brito-Loeza and K. Chen, Multigrid method for a modified curvature driven diffusion model for image inpainting, J. Comput. Math., 26(6), 856-875, 2008.

[19] M. Burger, L. He and C. Schönlieb, Cahn-Hilliard inpainting and a generalization for grayvalue images, SIAM J. Imaging Sci., 2 (4), 1129-1167, 2009.

[20] F. Cao, Y. Gousseau, S. Masnou and P. Perez, Geometrically guided exemplar-based inpainting, INRIA preprint, hal-00380394, 2009..

[21] V. Caselles, J.M. Morel, C. Sbert and A. Gillette, An axiomatic approach to image interpolation, IEEE Trans. on Image Proc., 7(3), 376-386, 1998.

[22] A. Chambolle, An algorithm for total variation minimization and applications, J. Math. Imaging Vis., 20(1-2), 89-97, 2004.

[23] T.F. Chan and J. Shen, Mathematical models for local non-texture inpaintings, SIAM J. Appl. Math., 62(3), 1019-1043, 2001.

[24] T.F. Chan and J. Shen, Non-texture inpainting by curvature driven diffusions (CDD), J. Visual Commun. Image Rep., 12(4), 436-449, 2001.

[25] T.F. Chan and J. Shen, Variational restoration of non-flat image features: models and algorithms, SIAM J. Appl. Math., 61(4), 1338-1361, 2001.

[26] T.F. Chan and J. Shen, Variational image inpainting, Commun. Pure Applied Math., 58, 579-619, 2005.

[27] T.F. Chan, S.H. Kang and J. Shen, Euler's elastica and curvature-based inpainting, SIAM J. Appl. Math., 63(2), 564-592, 2002.

[28] T.F. Chan, J.H. Shen and H.M. Zhou, Total variation wavelet inpainting, J. Math. Imaging Vis., 25(1), 107-125, 2006.

[29] A. Criminisi, P. Perez and K. Toyama, Object removal by exemplar-based inpainting, IEEE Int. Conf. Comp. Vision and Pattern Recog. 2, 721-728, 2003.

[30] J.A. Dobrosotskaya and A.L. Bertozzi, A Wavelet-laplace variational technique for image deconvolution and inpainting, IEEE Trans. Imag. Proc., 17(5), 657-663, 2008.

[31] J. Jr. Douglas and T. Dupont, Alternating-direction Galerkin methods on rectangles numerical solution of partial differential equations, II (SYNSPADE 1970) (Proc. Symp., University of Maryland, College Park, Md. 1970) (New York: Academic), 133-214, 1971.

[32] A.A. Efros and T.K. Leung, Texture Synthesis by Non-parametric Sampling, IEEE International Conference on Computer Vision, Corfu, Greece, September, 1999.

[33] C.M. Elliott and S.A. Smitheman, Analysis of the TV regularization and $H^{-1}$ fidelity model for decomposing an image into cartoon plus texture, Commun. Pure Appl. Anal., 6(4), 917-936, 2007.

[34] C.M. Elliott and S.A. Smitheman, Numerical analysis of the TV regularization and $H^{-1}$ fidelity model for decomposing an image into cartoon plus texture, IMA Journal of Numerical Analysis, 1-39, 2008.

[35] S. Esedoglu and J.H. Shen, Digital inpainting based on the Mumford-Shah-Euler image model, 
Eur. J. Appl. Math., 13(4), 353-370, 2002.

[36] D. Eyre, An unconditionally stable one-step scheme for gradient systems, Jun., 1998, unpublished.

[37] X. Feng and A. Prohl, Error analysis of a mixed finite element method for the Cahn-Hilliard equation, Numer. Math., 99(1), 47-84, 2004.

[38] X. Feng and A. Prohl, Numerical analysis of the Cahn-Hilliard equation and approximation for the Hele-Shaw problem, Interfaces Free Bound., 7(1), 1-28, 2005.

[39] M. Fornasier and C.B. Schönlieb, Subspace correction methods for total variation and $l_{1}-$ minimization, SIAM J. Numer. Anal., 47(5), 3397-3428, 2009.

[40] K. Glasner, A diffuse interface approach to Hele-Shaw flow, Nonlinearity, 16, 1-18, 2003.

[41] T. Goldstein, X. Bresson and S. Osher, Geometric applications of the split Bregman method: Segmentation and surface reconstruction, J. Sci. Comp., 45(1-3), pp. 272-293, 2010.

[42] T. Goldstein and S. Osher, The split Bregman method for L1 regularized problems, SIAM Journal on Imaging Sciences, 2(2), 323-343, 2009.

[43] J.B. Greer and A. Bertozzi, $H^{1}$ solutions of a class of fourth order nonlinear equations for image processing, Discrete Contin. Dyn. Syst., 10(1-2), 349-366, 2004.

[44] J.B. Greer and A. Bertozzi, Traveling wave solutions of fourth order PDEs for image processing, SIAM J. Math. Anal., 36(1), 38-68, 2004.

[45] H. Grossauer and O. Scherzer, Using the complex Ginzburg-Landau equation for digital inpainting in 2D and 3D, Scale Space Methods in Computer Vision, 2695, 225-236, 2003.

[46] G. Grün and M. Rumpf, Nonnegativity preserving convergent schemes for the thin film equation, Num. Math., 87, 113-152, 2000.

[47] J.W. Gu, L. Zhang, G.Q. Yu, Y.X. Xing and Z.Q. Chen, X-ray CT metal artifacts reduction through curvature based sinogram inpainting, Journal of X-Ray Science and Technology, 14(2), 73-82, 2006.

[48] M. Hirsch and S. Smale, Differential Equations, Dynamical Systems and Linear Algebra, Academic Press, London, 1974.

[49] L. Lieu and L. Vese, Image restoration and decompostion via bounded total variation and negative Hilbert-Sobolev spaces, Applied Mathematics \& Optimization, 58, 167-193, 2008.

[50] M. Lysaker, A. Lundervold and X.C. Tai, Noise removal using fourth-order partial differential equations with applications to medical magnetic resonance images in space and time, IEEE Trans. on Image Proc., 12(12), 1579-1590, 2003.

[51] O.M. Lysaker and X.-C. Tai, Iterative image restoration combining total variation minimization and a second-order functional, Inter. J. Comput. Vis., 66(1), 5-18, 2006.

[52] S. Masnou and J. Morel, Level lines based disocclusion, 5th IEEE Int'l Conf. on Image Processing, Chicago, IL, Oct., 4-7, 259-263, 1998.

[53] L. Modica and S. Mortola, Il limite nella $\Gamma$-convergenza di una famiglia di funzionali ellittici, Boll. Unione Mat. Ital., V. Ser., A14, 526-529, 1977.

[54] L. Modica and S. Mortola, Un esempio di $\Gamma^{-}$-convergenza, Boll. Unione Mat. Ital., V. Ser., B14, 285-299, 1977.

[55] M.J. Narashima and A.M. Peterson, On the computation of the discrete cosine transform, IEEE Trans. Commun. COM, 26, 934-936, June, 1978.

[56] L.K. Nielsen, X.C. Tai, S.I. Aanonsen and M. Espedal, Noise Removal of Seismic Data Using a Fourth-Order Parabolic PDE, International J. Tomography \& Statistics (IJTS), 4(6), 63, 2006.

[57] M. Nitzberg, D. Mumford and T. Shiota, Filtering, Segmentation, and Depth, Springer-Verlag, Lecture Notes in Computer Science, 662, 1993.

[58] S. Osher, A. Sole and L. Vese, Image decomposition and restoration using total variation minimization and the $H^{-1}$ norm, Multiscale Modeling and Simulation: A SIAM Interdisciplinary Journal, 1(3), 349-370, 2003.

[59] P. Perona and J. Malik, Scale-space and Edge Detection Using Anisotropic Diffusion, IEEE Transactions on Pattern Analysis and Machine Intelligence, 12(7), 629-639, July, 1990.

[60] L. I. Rudin, S. Osher and E. Fatemi, Nonlinear total variation based noise removal algorithms, Physica D, 60, 259-268, 1992.

[61] L. Rudin and S. Osher, Total variation based image restoration with free local constraints, Proc. 1st IEEE ICIP, 1, 31-35, 1994.

[62] C.B. Schönlieb, Total variation minimization with an $H^{-1}$ constraint, CRM Series 9, Singularities in Nonlinear Evolution Phenomena and Applications Proceedings, Scuola Normale Superiore Pisa, 2009, 201-232.

[63] C.B. Schönlieb, Modern PDE Techniques for Image Inpainting, PhD Thesis, University of Cambridge, 265, June 2009.

[64] C.B. Schönlieb, A. Bertozzi, M. Burger and L. He, Image Inpainting Using a Fourth-Order 
Total Variation Flow, Proc. Int. Conf. SampTA09, Marseilles, 2009.

[65] P. Smereka, Semi-implicit level set methods for curvature and surface diffusion motion, Special issue in honor of the sixtieth birthday of Stanley Osher, J. Sci. Comput., 19(1-3), 439-456, 2003.

[66] A.M. Stuart and A.R. Humphries, Model problems in numerical stability theory for initial value problems, SIAM Rev., 36(2), 226-257, 1994.

[67] R. Temam, Infinite-Dimensional Dynamical Systems in Mechanics and Physics, Appl. Math. Sci., Springer, New York, 68, 1988.

[68] A. Tsai, J.A. Yezzi and A.S. Willsky, Curve evolution implementation of the Mumford-Shah functional for image segmentation, denoising, interpolation and magnification, IEEE Trans. Image Process, 10(8), 1169-1186, 2001.

[69] J. Tumblin and G. Turk, LCIS: A Boundary hierarchy for detail-preserving contrast reduction, Siggraph, Computer Graphics Proceedings, 83-90, 1999.

[70] L. Vese and S. Osher, Modeling textures with total variation minimization and oscillating patterns in image processing, J. Sci. Comput., 19(1-3), 553-572, 2003.

[71] B.P. Vollmayr-Lee and A.D. Rutenberg, Fast and accurate coarsening simulation with an unconditionally stable time step, Phys. Rev. E, 68(0066703), 1-13, 2003.

[72] L.Y. Wei and M. Levoy, Fast texture synthesis using tree-structured vector quantization, In Proc. SIGGRAPH'00, New Orleans, USA, 479-488, 2000.

[73] A.L. Yuille and A. Rangarajan, The Concave-Convex procedure (CCCP), Neural Computation, 15(4), 915-936, April, 2003. 